\title{
Surface drifter pair spreading in the North Atlantic
}

\author{
Rick Lumpkin $^{1}$ and Shane Elipot ${ }^{2}$
}

Received 14 April 2010; revised 19 August 2010; accepted 17 September 2010; published 7 December 2010.

[1] This study examines spreading of surface drifter pairs deployed as part of the CLIVAR Mode Water Dynamic Experiment (CLIMODE) project in the Gulf Stream region. The spreading is resolved at hourly resolution and quantified by relative dispersion and finite-scale Lyapunov exponents. At scales from 1-3 km to $300-500 \mathrm{~km}$, the dispersion follows Richardson's law, indicating stirring by eddies comparable in scale to the pair separation distance. At larger scales, the spreading becomes a random walk described by a constant diffusivity. The behavior from $1-3 \mathrm{~km}$ to the local deformation radius is inconsistent with the enstrophy cascade of 2-D quasigeostrophic turbulence. To test various hypotheses for this result, drifter pair spreading is examined for pairs that were not launched together, pairs deployed in the eastern subtropical North Atlantic, and CLIMODE pairs subsampled to daily temporal resolution. Our results indicate the presence of significant energy at the submesoscale in the Gulf Stream region which flattens the wave number spectrum and dominates surface stirring at this scale range. Results in the less energetic subtropical eastern Atlantic are more equivocal.

Citation: Lumpkin, R., and S. Elipot (2010), Surface drifter pair spreading in the North Atlantic, J. Geophys. Res., 115, C12017, doi:10.1029/2010JC006338.

\section{Introduction}

[2] A large number of in situ oceanic studies have investigated ocean dispersion in the context of single-particle dispersion theory [Taylor, 1921]. This theory describes how a tracer cloud spreads from its release point as a function of the eddy fluctuations in a turbulent flow field, characterized by a Lagrangian length scale. If the position of a particle in the cloud is $x(t)$, then the cloud's spreading can be quantified by the absolute dispersion $\left\langle x^{2}\right\rangle$, where $\langle\cdot\rangle$ indicates an ensemble average over the particles. Alternatively, by analogy with molecular diffusion [Taylor, 1921; Davis, 1982], the spreading can be expressed as an effective diffusivity $\kappa=\frac{1}{2} \frac{\mathrm{d}}{\mathrm{d} t}\left\langle x(t)^{2}\right\rangle$. If the turbulent field is stationary and homogeneous then the effective diffusivity asymptotes to a constant value after the particle has traveled a distance exceeding the largest eddies in the turbulent field (which are generally larger than the characteristic Lagrangian length scale). This asymptotic behavior is the random walk regime [Taylor, 1921]. Before this, the effective diffusivity grows with time, proportional to time at very short times (the ballistic regime) then transitioning from ballistic toward random walk behavior at intermediate times.

[3] While single-particle dispersion quantifies the absolute rate of a cloud spreading at large scales, it does not characterize the spreading of particles about the center of mass of a cloud at small or intermediate scales. Furthermore,

\footnotetext{
${ }^{1}$ NOAA Atlantic Oceanographic and Meteorological Laboratory, Miami, Florida, USA.

${ }^{2}$ National Oceanography Centre, Liverpool, UK.

Copyright 2010 by the American Geophysical Union. 0148-0227/10/2010JC006338
}

focusing on a single length scale to characterize the turbulent flow neglects the fact that the flow is characterized by a range of scales. The amount of energy at a particular scale determines how a tracer cloud will be stirred relative to its center of mass at that scale. This stirring can be quantified by observing the spreading of pairs within the cloud. For example, consider a pair of drifters separated by a distance $d(t)$. The rate of change of $d(t)$ can be dominated by eddies of comparable scale if they have sufficient energy, because smaller eddies add a relatively weak random walk and larger eddies move the pair as a coherent unit to lowest order [Corrsin, 1962]. Thus, the spreading rate of particle pairs is sensitive to the distribution of energy as a function of length scale in the turbulent flow, i.e., the slope of the Eulerian wave number spectrum. Under the assumption of two-dimensional, stationary turbulence with velocity wave number spectrum $E(k) \propto k^{-\alpha}$, it can be shown [see Morel and Larcheveque, 1974; LaCasce, 2008] that

$$
\frac{1}{2} \frac{\mathrm{d}}{\mathrm{d} t}\left\langle d^{2}\right\rangle \propto d^{\frac{\alpha+1}{2}} \text { for } 1<\alpha<3 .
$$

This is the range in which pair spreading is primarily driven by eddies of the same scale as the separation of the pair, a condition known as "local dispersion" [Bennett, 1984]. However, if the wave number spectrum is steeper $(\alpha \geq 3)$, pair spreading is dominated by eddies larger than the separation distance; the separation grows exponentially, i.e., $\left\langle d^{2}\right\rangle \propto \exp (t)$ [Bennett, 1984], and the spreading is "nonlocal".

[4] Richardson [1926] first characterized turbulent stirring by quantifying particle pair separation. His observations included smoke spreading from a stack, seeds and balloons released into the air, and later [Richardson and Stommel, 
1948] floating pieces of parsnip dropped from a pier. Richardson [1926] proposed that $\left\langle d^{2}\right\rangle \propto t^{3}$, indicating that the rate of separation $\frac{\mathrm{d}}{\mathrm{d} t} d$ increases with increasing separation. This implies that the relative diffusivity $K=\frac{1}{2} \frac{\mathrm{d}}{\mathrm{d} t}\left\langle d^{2}\right\rangle$, the two-particle analogue of the single-particle absolute diffusivity, is scale dependent: $K \propto d^{4 / 3}$, a relationship now known as Richardson's law. Richardson's law behavior persists until $d$ exceeds the largest eddies in the turbulent field (if such a limit exists). Past that, the separation continues as a random walk $\left\langle d^{2}\right\rangle \propto t$, with constant $K$ equal to twice the single-particle diffusivity $\kappa$ [Richardson, 1926].

[5] Richardson [1926] was instrumental in characterizing three-dimensional turbulence as the superposition of eddies with a range of scales, and with energy cascading from the largest eddies to the scale of molecular dissipation. Assuming that the small-scale flow is homogeneous and isotropic, with statistics set by the molecular diffusion and the rate of energy dissipation, Kolmogorov [1941] showed that the velocity wave number spectrum in this "inertial" scale range must be $E(k) \propto k^{-5 / 3}$. Then, from (1), the relative diffusivity is $K \propto d^{4 / 3}$, i.e., Richardson's law.

[6] In the quasigeostrophic (QG) limit, motion at scales large enough to feel the vertical boundaries (2-D barotropic motion) exhibit the same wave number slope as 3-D turbulence at scales from the Rossby radius of deformation $L_{d}$ up to the largest scales of the turbulent flow [Kraichnan, 1967], although the energy cascade across this range is from smaller to larger scales, opposite to the energy cascade in 3-D turbulence. At scales smaller than $L_{d}$, 2-D QG turbulence has a second inertial range in which enstrophy (squared vorticity) cascades downscale and the velocity spectral slope is far steeper, $E(k) \propto k^{-3}$ [Kraichnan, 1967; Charney, 1971]. In this enstrophy cascade range, particle pair spreading is controlled by the rate of strain of the large, energy-containing eddies in the energy cascade range [Kraichnan, 1967], i.e., the dispersion is weakly nonlocal [Bennett, 1984], and particle pair separation is exponential. Thus, one expects particle pairs in 2-D QG turbulence to separate exponentially while their separation distance is smaller than $L_{d}$, then follow Richardson's law at separation distances from $L_{d}$ to the largest eddies in the flow. At larger separation distances, the separation should revert to a random walk characterized by a constant diffusivity.

[7] More recently, focus has turned to the behavior of quasigeostrophic turbulence in the presence of a boundary such as the ocean surface. The framework of this surface quasigeostrophic (SQG) theory was developed by Blumen [1978]. Away from the surface boundary, SQG can revert to 2-D geostrophic turbulence at large scales, in which the flow is driven by large-scale potential vorticity (PV) variations (in its simplest formulation, SQG has constant PV in the interior and the flow is limited to the surface). At the surface boundary, SQG dynamics are driven by lateral variations in density anomaly [Blumen, 1978]. These variations are not constrained by the vertical velocity (as in the interior), and thus SQG flow generates sharp vorticity gradients at the surface. These gradients in turn lead to energy injection at submesoscales, and create an inverse energy cascade from the submesoscale through the mesoscale [Capet et al., 2008]. This surface boundary layer flow dominates the interior QG flow at the surface for baroclinically unstable flow forced by the large-scale density gradient [Lapeyre and Klein, 2006]. SQG has been shown to describe the surface flow in numerical simulations of the North Atlantic where mesoscale activity is large (as in the Gulf Stream region) and where the mixed layer is deep [Isern-Fontanet et al., 2008]. The surface mode of SQG dominates North Atlantic simulations in the Gulf Stream and North Atlantic Drift regions, while the interior first baroclinic mode is dominant in less energetic regions such as the recirculating Azores Current region [Lapeyre, 2009]. As a consequence of SQG dynamics, the velocity wave number spectral slope at scales smaller than the mesoscale is much shallower than in the enstrophy cascade range of 2-D QG turbulence, with a slope of $k^{-5 / 3}$ [Blumen, 1978] for linear (purely geostrophic) SQG. Extremely high-resolution primitive equation simulations, which can ignore the $\mathrm{QG}$ assumption of small Rossby number flow, reproduce several key predictions of SQG theory including a flattening of the near-surface velocity wave number slope for a range of scales smaller than $L_{d}$ [Klein et al., 2008]. Thus, SQG theory predicts Richardson's law behavior for the relative dispersion from the submesoscale, $\mathrm{O}(1 \mathrm{~km})$, to the scale at which the bottom boundary is felt by the flow and it behaves like 2-D turbulence [Tulloch and Smith, 2006]. An extension of SQG theory that includes two active interior modes [Tulloch and Smith, 2009] demonstrates that the transition from SQG to 2-D QG turbulence occurs at the deformation radius $L_{d}$ when the near-surface shear of the flow greatly exceeds the magnitude of the speed divided by the fluid depth. This is generally the case in the ocean, but not in the atmosphere where the transition occurs at smaller scales [Tulloch and Smith, 2009]. At larger scales, Richardson's law behavior is consistent with 2-D QG turbulence as noted above.

[8] The presence of large-scale mean shear can complicate the interpretation of dispersion. For example, [Bennett, 1987; LaCasce, 2008], in the presence of a meridionally sheared zonal flow $U(y)$, a pair with meridional separation $d_{y}$ will spread in the zonal direction according to

$$
<d_{x}^{2}>=\left(\frac{\mathrm{d} U}{\mathrm{~d} y}\right)^{2}<d_{y}^{2}>t^{2}
$$

If the meridional spreading is a random walk, i.e., $\left\langle d_{y}^{2}\right\rangle \propto t$, then the zonal dispersion will be proportional to $t^{3}$ (Richardson's law). Shear dispersion is highly anisotropic, and the zonal and meridional separation rates is correlated while (in this simple example) the zonal and meridional eddy speeds of the two particles are uncorrelated at all scales.

[9] There are relatively few observational studies to assess the disparate predictions of 2-D QG turbulence and SQG theory at subdeformation radius scales. Altimetry-derived sea height anomaly spectral slopes are flatter than predicted by 2-D QG turbulence, and are instead consistent with SQG theory [Le Traon et al., 2008]. However, velocity spectra calculated from repeated ADCP crossings of the Gulf Stream exhibit a $k^{-3}$ slope, consistent with 2-D QG turbulence [Wang et al., 2010]. Qualitatively, observations of submesoscale vortex merging [Flament et al., 2001] imply an inverse kinetic energy cascade at the submesoscale, as predicted by SQG. In situ relative dispersion studies are rare. LaCasce and Bower [2000] examined subsurface floats from several experiments in the eastern and western North 
Atlantic, but were forced to rely on pairs that encountered each other by chance (a constraint that severely reduces the ability to resolve relative dispersion at submesoscales). Floats in the eastern Atlantic did not demonstrate correlated velocities at the closest resolvable distance of $\mathrm{O}(10 \mathrm{~km})$, and thus measured absolute (single particle) dispersion, with random walk behavior at separations greater than $\sim 50 \mathrm{~km}$. The western float pairs of LaCasce and Bower [2000] had correlated velocities for separation distances from $10 \mathrm{~km}$ up to $\sim 100 \mathrm{~km}$, and exhibited Richardson's law behavior in this range. The dispersion was isotropic across all scales. At separations greater than $200 \mathrm{~km}$, the western float pairs dispersed in a random walk. More recently, Ollitrault et al. [2005] examined subsurface float pairs in the western and eastern North Atlantic with initial separation of a few kilometers, and found early, brief exponential separation for their 16 eastern float pairs. All floats exhibited Richardson's law dispersion for 40-300 km separation, and random walk dispersion at larger separation. Exponential separation at scales smaller than the Rossby radius is consistent with QG turbulence. It should be noted that intermediate to deep subsurface float dispersion wouldn't be affected by the $\mathrm{O}(250 \mathrm{~m})$ ocean surface boundary, and should thus behave according to standard QG theory. In contrast, surface drifters should feel the boundary flow of SQG theory, where that theory applies.

[10] The most comprehensive study of surface drifter relative dispersion is LaCasce and Ohlmann [2003]. LaCasce and Ohlmann [2003] examined the spreading rate of drifter pairs and triplets gathered from three extremely dense arrays of Coastal Ocean Dynamics Experiment-type (also known as Davis-type) drifters deployed in the Gulf of Mexico, north and west of the energetic Loop Current. These drifters were tracked by Argos positioning [see Lumpkin and Pazos, 2007], which, prior to January 2005, provided 5-7 position fixes per day. These fixes were interpolated to daily resolution [LaCasce, 2008] for their dispersion calculations. Few of the drifters were deployed as pairs, so LaCasce and Ohlmann [2003] had to rely heavily upon "chance pairs": pairs that happened to come close to each other. LaCasce and Ohlmann [2003] found that the drifter pairs separated exponentially from the smallest resolvable distance of $\mathrm{O}(1 \mathrm{~km})$ to $40-50 \mathrm{~km}$, the local deformation radius, consistent with the predictions of 2-D QG turbulence in the enstrophy cascade inertial subrange. At larger separations, the pairs spread at a rate consistent with Richardson's law and QG turbulence in the inverse energy cascade subrange.

[11] LaCasce and Ohlmann [2003] stated that they found a consistent exponential spreading rate at subdeformation radius scales using two methods: relative dispersion and finite-scale Lyapunov exponents (FSLEs, described in detail later in this paper), with the transition from exponential to Richardson's law behavior happening near the deformation radius in both methods. However, more recently LaCasce [2008] raised doubt on this conclusion. He noted that the spreading rates were not consistent between these two methods, since LaCasce and Ohlmann [2003] compared a distance-squared rate from relative dispersion to a distance rate from the FSLEs. He also found that the temporal resolution of the data strongly affected the FSLE results, and that smooth interpolation of the data to increasingly higher temporal resolution shifted the apparent transition from exponential to Richardson's law behavior to increasingly smaller scales [LaCasce, 2008].

[12] Most recently, Koszalka et al. [2009] examined the dispersion of a large number of drifter pairs in the Nordic Seas, most of which were launched together. They found exponential separation up to the local deformation radius, and then separation consistent with Richardson's law to $\sim 100 \mathrm{~km}$. However, because of the small Rossby radius $(10 \mathrm{~km})$ in that region, the pairs reached the transition from exponential to Richardson spreading in a relatively brief 2 days.

[13] In this study, we examine the spreading of 55 drifter pairs deployed in tight clusters in the Gulf Stream, in February-March 2007, as part of the CLIVAR Mode Water Dynamics Experiment (CLIMODE). The drifters' spreading was resolved at approximately hourly resolution. At scales from $\sim 1-3 \mathrm{~km}$ to $300-500 \mathrm{~km}$, we find dispersion exhibiting Richardson's law behavior, while at larger scales the pair spreading is consistent with a single-particle constant effective diffusivity of $\mathrm{O}\left(1-2 \times 10^{4} \mathrm{~m}^{2} \mathrm{~s}^{-1}\right)$. The behavior from $1-3 \mathrm{~km}$ to the first baroclinic Rossby radius of $30 \mathrm{~km}$ [Chelton et al., 1998] is qualitatively different than the Gulf of Mexico drifters described by LaCasce and Ohlmann [2003] and Koszalka et al. [2009], i.e., not separating exponentially before reaching the deformation radius. To explore why we find this subdeformation-scale behavior, we also examine the spreading of drifter pairs away from the Gulf Stream region, the spreading of Gulf Stream chance pairs in the historical drifter data set, and the effect of lowered temporal resolution on our calculations. We conclude that drifters in the Gulf Stream region do not exhibit exponential separation at scales larger than $1-3 \mathrm{~km}$. We interpret this as evidence of significant energy input at the submesoscale which flattens the wave number spectrum. This behavior is consistent with the predictions of surface quasigeostrophic turbulence theory. We also find that reduced temporal resolution can contaminate the FSLE calculations and produce (spurious) exponential separation at the submesoscale. For drifter pairs in the more quiescent eastern subtropical Atlantic, we find more equivocal results: relative dispersion at scales smaller than the Rossby radius is extremely noisy, with large error bars, but is better explained by exponential separation than by Richardson's law behavior. In contrast, the FSLE results from the eastern drifter pairs indicate that the pairs separate according to Richardson's law at the submesoscale.

\section{Data}

[14] All observations analyzed in this study were collected by satellite-tracked surface drifters, drogued at $15 \mathrm{~m}$ to follow near-surface currents [Niiler, 2001; Lumpkin and Pazos, 2007]. Since January 2005 multisatellite tracking has provided a location fix for each drifter every 1-2 h [Elipot and Lumpkin, 2008]. These position fixes are rated according to "location class": class 3 (uncertainty $<150 \mathrm{~m}$ ), class $2(150-300 \mathrm{~m})$, class $1(350-1000 \mathrm{~m})$, and class 0 (uncertainty $>1 \mathrm{~km}$ ). In this study, no class 0 fixes were used.

[15] All drifters include either a submergence or a tether strain sensor for determining drogue presence. These data 


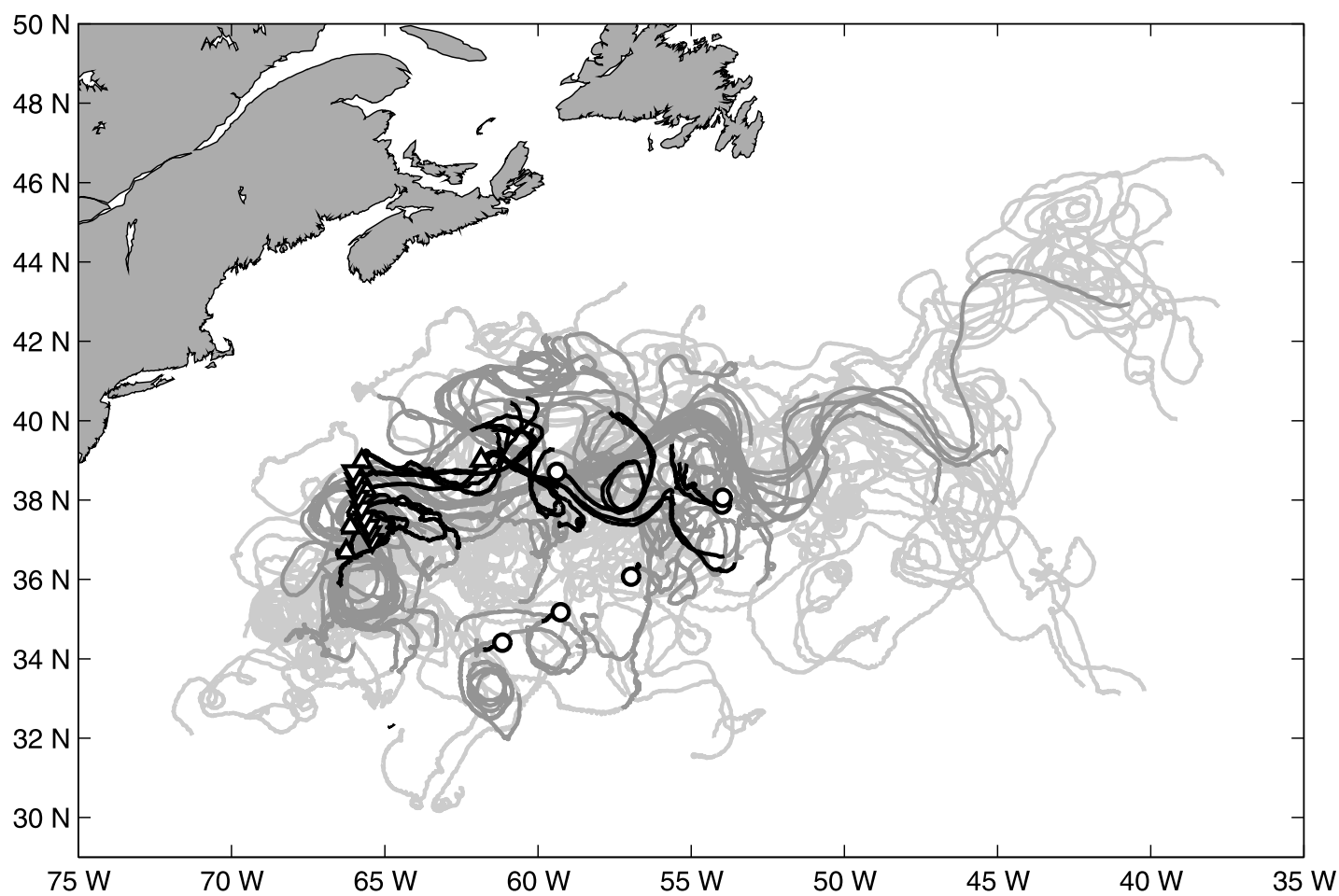

Figure 1. The CLIMODE drifter array. Deployment positions are shown for pairs (circles) and trios during leg 1 of the cruise (February 2007) and trios during leg 2 (March 2007; inverted triangles). Subsequent drifter trajectories are shown in black (February), dark gray (March), and light gray (1 April to 7 July, the final data analyzed here).

are used to determine the drogue off time for each drifters. Because undrogued drifters are subject to significant slip, i.e., motion relative to the current at $15 \mathrm{~m}$ [Niiler and Paduan, 1995], trajectories from drifters that had lost their drogues were not examined in this study.

\subsection{CLIMODE Drifter Array}

[16] As part of the CLIVAR Mode Water Dynamics Experiment [Marshall et al., 2009], an array of 60 drifters was deployed in the Gulf Stream and its anticyclonic recirculation in February-March 2007 from the R/V Knorr (Figure 1). The overall goal of CLIMODE is to better understand the processes governing the formation and evolution of North Atlantic subtropical mode water. The drifter array was intended to provide measurements of eddy stirring in the formation region of this mode water, and to provide in situ sea surface temperature and velocity measurements for calibration and validation of satellitebased products.

[17] The drifters were deployed in pairs or trios, each launched over the span of a few seconds with an initial separation of a few meters maximum. One of the drifters failed to transmit any data. The other 59 drifters provided continuous fixes with one exception, a gap of 17.2 days for one drifter after 3.7 days in the water. Neglecting this gap, the median, mean, and standard deviation of the temporal gap between fixes was $0.96,1.17$, and $1.55 \mathrm{~h}$, respectively; $23 \%$ of the fixes were class $3,44 \%$ were class 2 , and $33 \%$ were class 1 . Conservatively assuming that each fix had the largest possible error for its class, the overall mean error for all fixes was approximately $500 \mathrm{~m}$. If the error for each drifter in a pair is independent, then the separation distance had a mean error of $\sqrt{2} \times 500=700 \mathrm{~m}$. Separation distances smaller than this were not significantly different from zero. Of the 59 drifters in the array, 55 pairs had their earliest satellite position fixes placing them less than $700 \mathrm{~m}$ apart.

[18] We linearly interpolated the raw fixes of the drifters to regular hourly intervals at 0000 UTC, 0100 UTC, etc., with the 17.24 day gap left uninterpolated. Velocities were calculated by a centered $2 \mathrm{~h}$ difference. The center of mass of each drifter pair was calculated as the mean latitude and longitude of the two drifters in the pair.

\subsection{Chance Drifter Pairs in the Gulf Stream Region}

[19] Several previous studies such as LaCasce and Bower [2000] and LaCasce and Ohlmann [2003] relied heavily upon chance pairs. It is possible that, by selectively isolating regions of surface convergence or confluence, such pairs are not homogeneously sampling the turbulent field in a statistical sense [LaCasce and Ohlmann, 2003]. To evaluate how this might affect our results in the Gulf Stream region, we identified all drifter pairs in the region $\left(25-45^{\circ} \mathrm{N}, 40-\right.$ $80^{\circ} \mathrm{W}$ ) in the historical drifter data set that came within $10 \mathrm{~km}$ of each other by chance (Figure 2) since January 2005 , the introduction of multisatellite processing. Some of these pairs were not chance pairs, as they had been launched together; we identified and eliminated these nonchance pairs. A total of 29 chance pairs ( 58 drifters) came within $10 \mathrm{~km}$, but only nine pairs came within $700 \mathrm{~m}$, the threshold used for relative dispersion of the CLIMODE drifters. We used these nine pairs for relative dispersion 


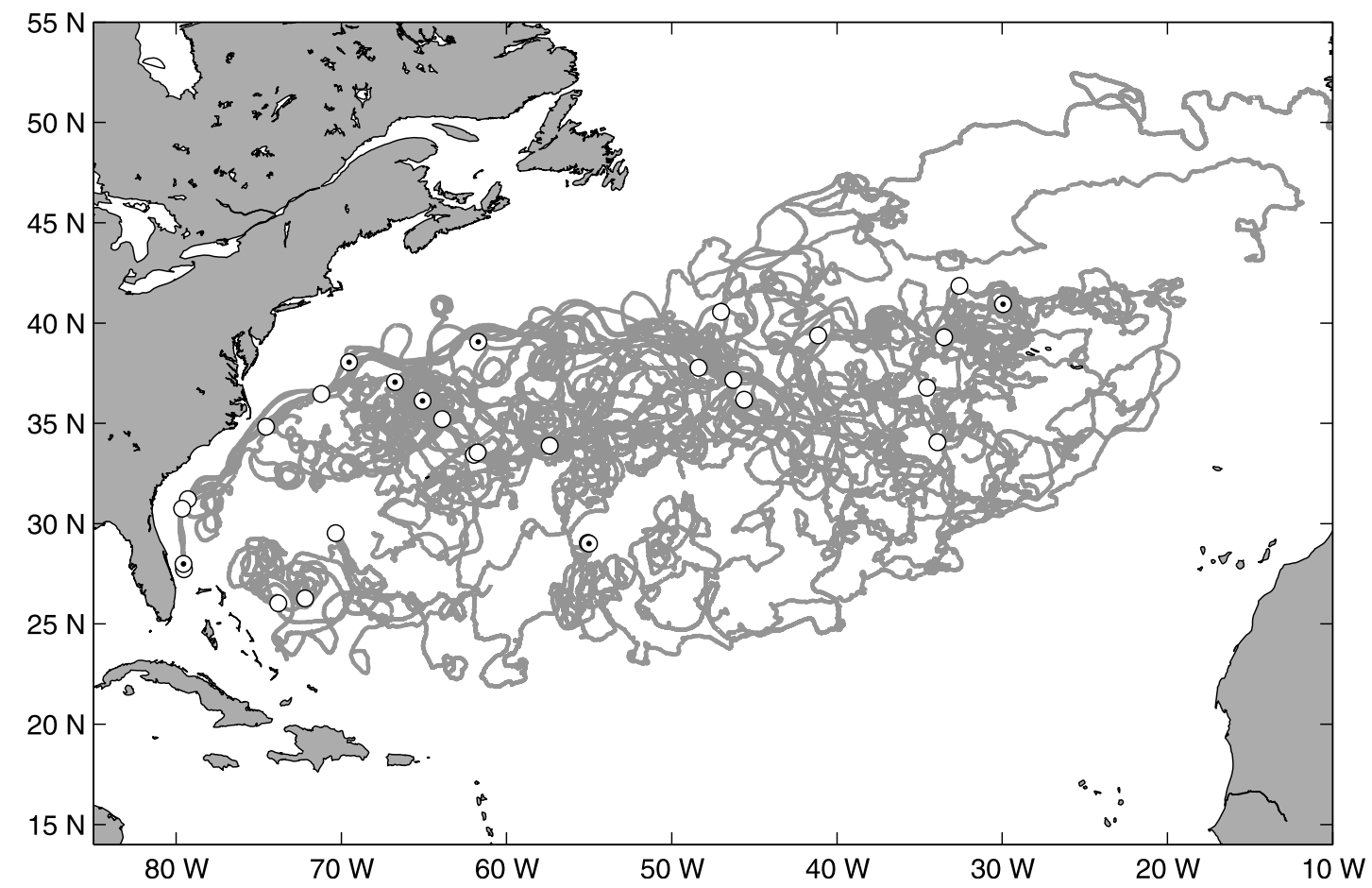

Figure 2. Location of 29 chance drifter pairs' closest approach points (circles) in the Gulf Stream region, 2005-2007. The nine pairs that came within $700 \mathrm{~m}$ of each other are indicated by black points (two pairs were in the Florida Current near $28^{\circ} \mathrm{N}$, and two were at $29^{\circ} \mathrm{N}, 55^{\circ} \mathrm{W}$ ). The trajectories of the 29 pairs are shown after the closest approach point.

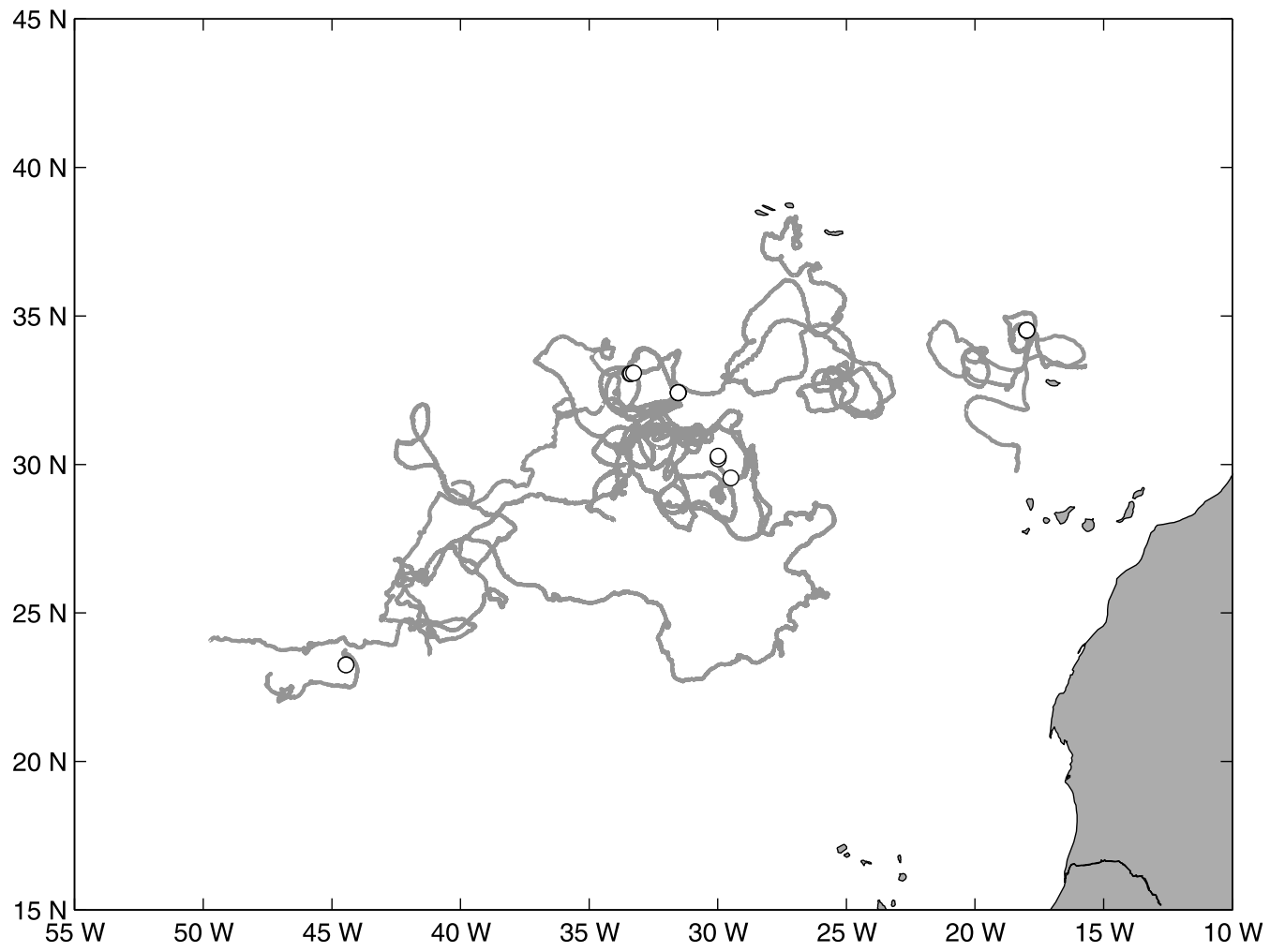

Figure 3. Location of 2005 and 2006 drifter cluster deployments (circles) and subsequent trajectories in the eastern subtropical North Atlantic. 
Table 1. Four-Drifter Clusters Launched in the Eastern Subtropical North Atlantic as Part of the ADB Evaluation Study ${ }^{\mathrm{a}}$

\begin{tabular}{lcccc}
\hline \multicolumn{1}{c}{ Date } & Latitude & Longitude & Number & Pairs \\
\hline 19 May 2005 & $33^{\circ} 3.8^{\prime} \mathrm{N}$ & $33^{\circ} 20.9^{\prime} \mathrm{W}$ & 3 & 1 \\
30 Aug 2005 & $23^{\circ} 15.1^{\prime} \mathrm{N}$ & $44^{\circ} 270^{\prime} \mathrm{W}$ & 2 & 1 \\
20 Sep 2005 & $34^{\circ} 31.2^{\prime} \mathrm{N}$ & $17^{\circ} 59.6^{\prime} \mathrm{W}$ & 4 & 6 \\
16 Mar 2006 & $30^{\circ} 41^{\prime} \mathrm{N}$ & $29^{\circ} 51.7^{\prime} \mathrm{W}$ & 4 & 4 \\
23 Jun 2006 & $32^{\circ} 25.0^{\prime} \mathrm{N}$ & $32^{\circ} 31.2^{\prime} \mathrm{W}$ & 4 & 6 \\
\hline
\end{tabular}

a"Date" is the deployment date. "Number" indicates the total number of drifters in the cluster that successfully transmitted data, with drogue attached. "Pairs" is the number of pairs with first position fixes placing them no further apart than $700 \mathrm{~m}$.

calculations, and all 58 drifters for calculating finite-scale Lyapunov exponents (see section 3).

\subsection{Drifter Pairs in the Eastern Subtropical North Atlantic}

[20] Although the primary focus of this study is the CLIMODE drifter array, we will seek additional insight on our results by comparing drifter pair spreading in the energetic Gulf Stream region to drifter pair spreading in the more quiescent eastern subtropical North Atlantic. In 2005-2006, a number of drifter clusters were launched throughout the Atlantic Ocean for the AOML Drifting Buoy (ADB) evaluation study. Each cluster consisted of four drifters, one from each of the four major drifter manufacturers, each with the same nondimensional drag area ratio that determines their water-following characteristics [Niiler et al., 1987]. The drifters in each cluster were launched within a few meters of each other. The purpose of the ADB study is to evaluate the packaging, activation, and postdeployment lifetime and behavior of the sensors, drogues and transmitters; the ability to examine pair separation is a side benefit.

[21] Six of these four-drifter clusters were launched in the eastern subtropical Atlantic $\left(22-35^{\circ} \mathrm{N}, 15-45^{\circ} \mathrm{W}\right)$ (Figure 3), each providing up to six possible drifter pairs (fewer pairs were realized in clusters where one or more drifters failed on deployment or indicated drogue lost upon deployment). Details of launch times, locations, and pairs are given in Table 1. A total of 18 drifter pairs had their first satellite fixes placing them within $700 \mathrm{~m}$ of each other, i.e., not significantly different from zero, and were used for calculating relative dispersion. All possible pairs were used for calculating finite-scale Lyapunov exponents.

\section{Methods}

[22] In this study, we shall quantify pair spreading using two methods: relative dispersion and finite-scale Lyapunov exponents.

\subsection{Relative Dispersion}

[23] Relative dispersion is the mean pair separation distance squared as a function of time

$$
D^{2}(t)=\left\langle d_{n}^{2}(t)\right\rangle,
$$

where $d_{n}$ is the separation distance of pair $n$ at time $t$ after release. In this study, only drifter pairs with an initial separation distance less than $700 \mathrm{~m}$ (the noise level of $d_{n}$ ) are used to calculate relative dispersion. This constraint imposes a strict limit on the number of pairs that can go into the calculation (Figure 4, top), but is necessary to resolve dispersion properly at small times after release. As time increases, the number of pairs going into the calculation will decrease due to drifters dying or losing their drogues.

[24] The effective relative diffusivity is given by the time rate of change of $D^{2}(t)$. Thus, the slope of $D^{2}(t)$ versus $t$ in a log-log plot indicates the dispersion regime experienced by the particles as they are stirred by the turbulent field. This dispersion regime is often observed to change with increasing time [see Taylor, 1921; LaCasce, 2008]. The diffusive regime is $D^{2}(t) \propto t$ (i.e., a constant effective diffusivity). "Ballistic" dispersion is $D^{2}(t) \propto t^{2}$. Richardson's law behavior is $D^{2}(t) \propto t^{3}$. The exponential separation regime is $D^{2}(t) \propto \exp (t)$.

\subsection{Finite-Scale Lyapunov Exponents}

[25] Finite-scale Lyapunov exponents are an alternative approach to examine particle pair separation. In this approach, one examines the growth of the ensembleaveraged separation distance $\delta$ (the use of $\delta$ rather than $d$ indicates that this distance is calculated as an ensemble average in spatial bins). This separation distance $\delta$ is
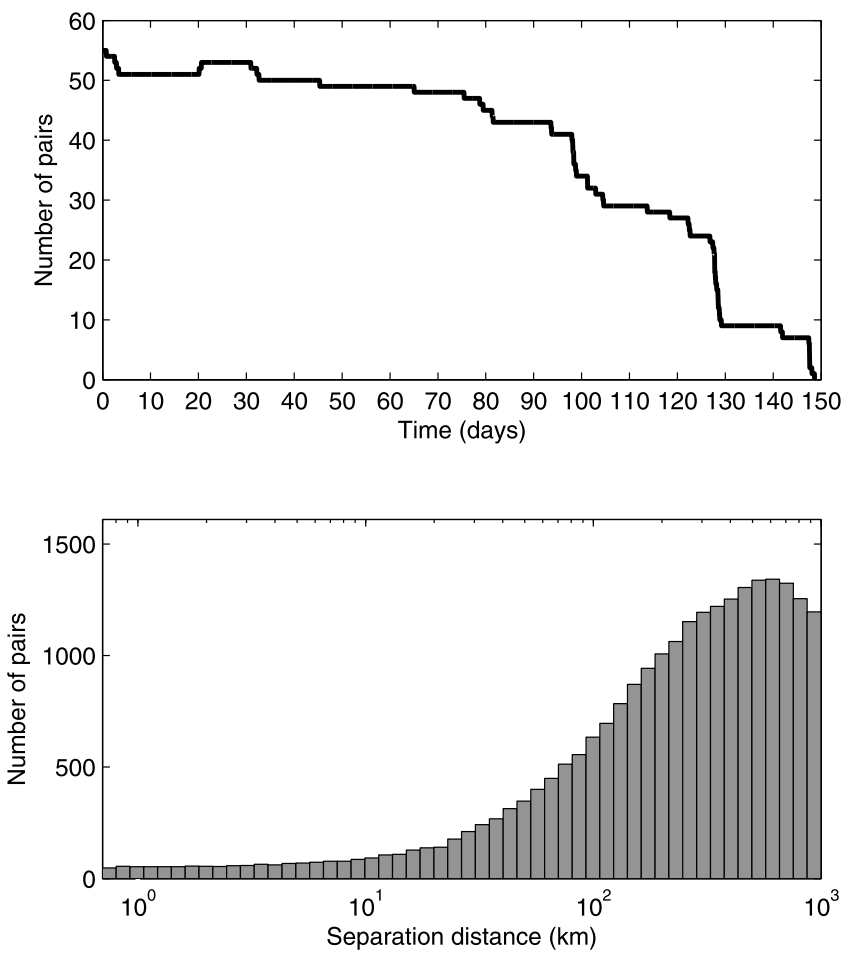

Figure 4. (top) Number of CLIMODE drifter pairs with initial separation distance less than $700 \mathrm{~m}$, as a function of time after deployment. Drifter deaths and drogue losses reduce the initial value, 55 pairs, to 26 pairs after 122 days and to 0 after 149 days. The increase at day 20 is caused by a drifter resuming transmissions after a gap of 17.2 days. (bottom) Number of pairs with separation distance falling within each distance bin $\left[\delta_{n}, \delta_{n+1}\right]$ used to calculate the finite-scale Lyapunov exponents. Note that a pair can provide more than one estimate of the FSLE within a bin (see Appendix A). 
quantified as $\delta=\delta_{0} \exp (\lambda t)$, i.e., exponential growth if the Lyapunov exponent $\lambda$ is constant [Artale et al., 1997; Aurell et al., 1997]. More generally, $\lambda$ can vary as a function of $\delta$. Over an interval $\left[\delta_{n}, \delta_{n+1}\right]$ in which $\lambda$ is approximately constant, $\left(t_{n+1}-t_{n}\right) \lambda=\ln \left(\delta_{n+1} / \delta_{n}\right)$. For $\delta_{n+1}=$ $\alpha \delta_{n}$, this becomes

$$
\lambda\left(\delta_{n}\right)=\frac{\ln \alpha}{\Delta t_{n}}
$$

where $\Delta t_{n}=t_{n+1}-t_{n}$ is the time for the separation distance to grow from $\delta_{n}$ to $\alpha \delta_{n}$. In practice, $\lambda$ is calculated by identifying $\Delta t_{n}$ for each pair with separation growing from $\delta$ to $\alpha \delta$, and averaging the right-hand side of (3) over all pairs. Because this calculation is conducted over a finite-scale range $(\alpha>1)$, it is known as the finite-scale Lyapunov exponent.

[26] Because $\lambda$ is an inverse time scale, the slope of $\lambda$ versus $\delta$ in a log-log plot can be related to the various dispersion regimes. In general, steeper slopes indicate slower dispersion. For Richardson's law behavior, one would expect $\lambda \propto \delta^{-2 / 3}$ from dimensional arguments [Haza et al., 2008]. For diffusive behavior, $\lambda \propto \delta^{-2}$. Steeper slopes are associated with subdiffusive behavior, which can be caused by particle trapping in coherent vortices [Provenzale, 1999], while constant $\lambda$ versus $\delta$ indicates exponential separation associated with chaotic advection [see Aref, 1984]. More generally, if the dispersion $D^{2} \propto t^{\alpha}$, then $\lambda \propto \delta^{-2 / \alpha}$ [LaCasce, 2008].

[27] The FSLE approach sorts the data by separation distance, rather than averaging in time as done when calculating relative dispersion. Thus, it is more appropriate if the processes governing pair spreading are scale dependent. Also, as a consequence of spatial averaging, all possible particle pairs can be included in the calculation, regardless of their minimum or initial separation. Thus, with increasing separation distance, an increasing number of pairs can be identified for calculating $\lambda$ (Figure 4 , bottom). This is in sharp contrast to relative dispersion, which relies upon a data set that decreases with increasing time (Figure 4, top) as drifters in the original pairs die or lose their drogues.

[28] However, there are some drawbacks to the FSLE approach. The results can be sensitive to data temporal resolution at small separation scales [LaCasce, 2008]. Also, there is not a consensus on how to calculate $\lambda$ for pairs when their separation distance does not grow monotonically. These issues, and the algorithm we use to implement (3), are described in detail in Appendix A.

\section{Results}

\subsection{Qualitative Assessment}

[29] The spreading of each drifter pair was examined visually, using weekly, delayed mode AVISO gridded sea height anomalies [Le Traon et al., 1998] superimposed on the mean dynamic height field of Rio and Hernandez [2004] to visualize the large-scale geostrophic velocity field. The optimally interpolated, merged TMI/AMSR-E microwave sea surface temperature product generated by Remote Sensing Systems was used to identify the location of the Gulf Stream front.
[30] At scales less than $\mathrm{O}(1 \mathrm{~km})$, there was obvious random noise dominating drifter pair separation, consistent with uncorrelated errors in the satellite-based location fixes. At larger scales, the separation of a pair was rarely monotonic, and was often characterized by extended periods of slow or negligible separation and shorter periods of very rapid separation, with occasional periods of convergence (decreasing separation).

[31] At scales larger than $\sim 10 \mathrm{~km}$, the pairs' center of mass tended to follow contours of absolute sea height. Rapid spreading events at separation distances $<10 \mathrm{~km}$ did not appear to be related to the sea height field. At larger separations, rapid spreading was rare and usually associated with the pair reaching a bifurcation point in the sea height field. For example, a few pairs spread to $10-20 \mathrm{~km}$ apart before reaching a saddle point between two highs in sea height, then diverged rapidly as they initiated orbits around these independent rings or meanders. However, at all scales of separation, many cases could be found where the separation was relatively steady and not obviously influenced by the geostrophic flow field.

[32] For the first 10 days after deployment, most pairs separated more slowly than the mean rate due to relatively rare rapid separation events. For example, 51 pairs with initial separation $<700 \mathrm{~m}$ were still transmitting 10 days later: their mean separation at 10 days was $50 \mathrm{~km}$, with $70 \%$ closer than $50 \mathrm{~km}, 50 \%$ closer than $30 \mathrm{~km}, 17 \%$ closer than $10 \mathrm{~km}$, and one pair was closer than $1 \mathrm{~km}$. In contrast, 9 of these pairs $(17 \%)$ were more than $100 \mathrm{~km}$ apart at 10 days. This asymmetry diminished after 11-13 days, with a comparable number of pairs above and below the mean separation distance at longer times. Most of the rapid separation events, but not all, occurred for pairs south of the Gulf Stream front. There was no clear relationship between the location of the front and the location of drifter pairs that spent an extended time at separation less than $5 \mathrm{~km}$.

[33] Most of the drifter pairs did not exhibit sustained spinning or looping about their center of mass (an exception is noted below). Occasional inertial oscillations were evident, as coherent anticyclonic looping of both drifters that did not significantly affect the pair separation.

[34] While space constraints prevent discussing every pair's spreading for an array of this size, a few cases highlight characteristics of pair spreading in the CLIMODE array: a "typical" pair, i.e., one that did not exhibit unusual spreading behavior, a pair that exhibited rapid spreading, and a pair that stayed close together for an anomalously long time.

\subsubsection{Typical Spreading Case}

[35] Drifters 72061 and 72072 (these are the identification numbers used in the Global Drifter Program database) were released just south of the Gulf Stream front on 4 March 2007. The pair drifted southward and began orbiting a cyclonic cold-core ring, visible in sea height (Figure 5a). During their first 4 days, the distance between them increased at approximately the mean rate, reaching $\sim 10 \mathrm{~km}$ after 4 days. This early spreading was not related to scales or features significantly larger than the separation distance, as resolved by sea height or SST. From days 6-9 after release, the drifter pair converged and diverged as the drifters orbited the ring, with separation distance fluctuating between 12 and $25 \mathrm{~km}$ as the drifters independently moved closer to the 


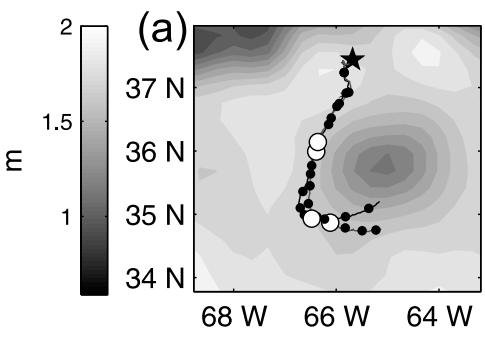

(b)
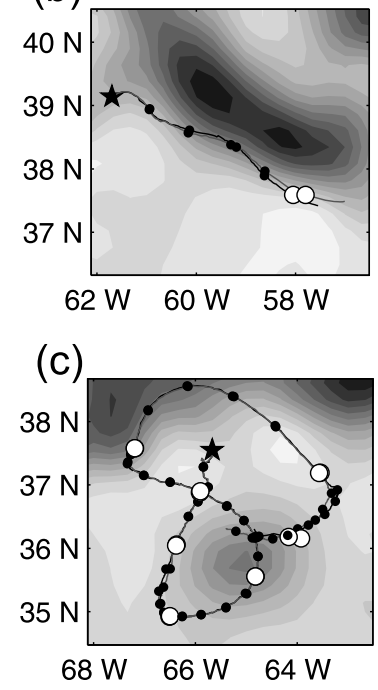
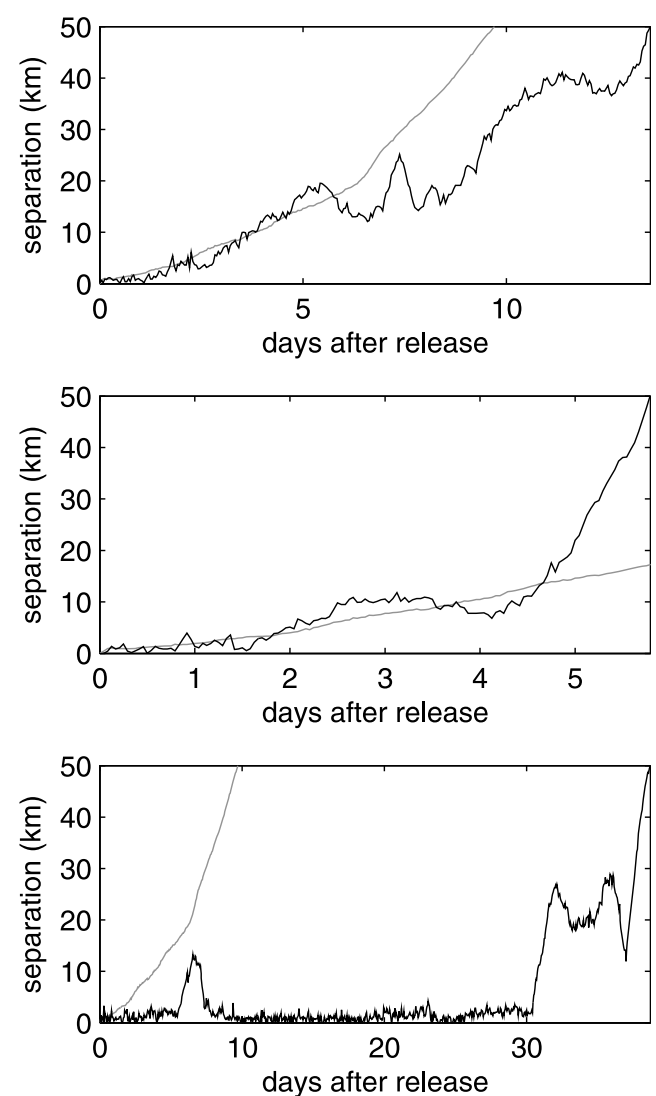

Figure 5. Examples of drifter pair spreading. (left) Drifter trajectories superimposed on absolute sea height (m), with drifter positions shown at daily (black dots) and 5 day (white circles) increments after release. Trajectories are shown from release (black stars) until the separation distance reached $50 \mathrm{~km}$. (right) Separation distance as a function of time for the drifter pair (black) and the mean separation distance versus time of all 55 pairs (gray). (a) Typical spreading case released on 4 March 2007, with sea height centered on 7 March. (b) Rapid spreading case released on 14 February, with sea height centered on 14 February. (c) Inhibited spreading case released on 4 March 2007, with sea height on 7 March.

ring center and accelerated, or further from the center and decelerated. Finally, after day 9, one drifter (72072) moved far enough from the ring to no longer feel its influence, while the other continued to orbit the ring. Over the next day, drifter 72072 moved westward, turned southward as 72061 looped northward, and the motion of the two drifters became uncorrelated thereafter.

\subsubsection{Rapid Spreading Case}

[36] Drifters 72055 and 72056 were released as part of a trio on 14 February, in the maximum surface velocity core of the Gulf Stream. Over the first 4.5 days, they moved at $90-110 \mathrm{~cm} \mathrm{~s}^{-1}$ while separating at very close to the average rate (Figure 5b). Starting on day 5, drifter 72055 shifted slightly south and slowed to a mean speed of $75 \mathrm{~cm} \mathrm{~s}^{-1}$ while 72056 , slightly to the north, continuing westward at a mean speed of $110 \mathrm{~cm} \mathrm{~s}^{-1}$. With a sustained velocity difference of $35 \mathrm{~cm} \mathrm{~s}^{-1}$, the separation distance rapidly increased. By day 7 the drifters were $70 \mathrm{~km}$ apart as the leading (with respect to the direction of propagation) drifter (72056) reached a sharp southward meander in the Gulf Stream. Their separation distance decreased to $34 \mathrm{~km}$ over the next day as the leading drifter negotiated the bend, then increased to more than $150 \mathrm{~km}$ (not shown in Figure 5) as the leading drifter continued southward along the front while the trailing drifter encountered the meander.

\subsubsection{Inhibited Spreading Case}

[37] Drifter 72054 was released as a trio with 72061 and 72072 , discussed in the "typical spreading case" above. However, this drifter and 72061 stayed extremely close together for 5.5 days (separation distance $<2 \mathrm{~km}$; Figure $5 \mathrm{c}$ ). From day 5.5 to 6.5 , the pair began separating at a rapid rate, reaching $13 \mathrm{~km}$ separation, but then reconverged to a separation distance often not distinguishable from zero due to position fix errors. After reconverging, the drifters stayed closer than $3 \mathrm{~km}$ together for a full 23 days, an exceptional and extremely anomalous case. On days $20-23$, the separation distance increased to $\sim 2 \mathrm{~km}$ and the drifters could clearly be seen orbiting their center of mass cyclonically (counterclockwise), with a period of $0.87-0.90$ days, completing three full orbits and beginning a fourth before reconverging to distances $<1 \mathrm{~km}$ (Figure 6). Orbiting could not be resolved when the pair was closer, due to position fix errors. From days 23-26, the drifters were closer than $1 \mathrm{~km}$. Separation distance increased again to $>2 \mathrm{~km}$ on day 26 , this time with no rotation about their center of mass. The drifters stayed $\sim 2 \mathrm{~km}$ apart until day 30 , when they abruptly 


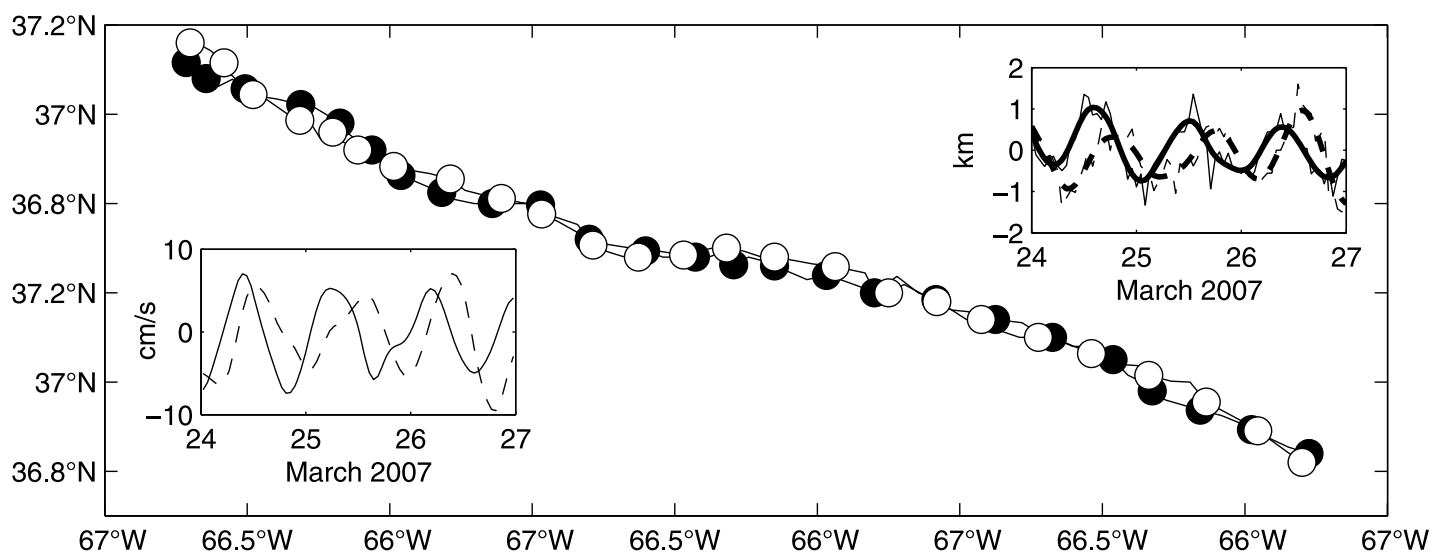

Figure 6. Locations of drifters 72054 (white) and 72061 (black) during 24-27 March 2007; positions are shown every $3 \mathrm{~h}$. During this time, the pair moved from bottom right to top left, while rotating cyclonically about their center of mass (CM). Inset at top right shows zonal (solid) and meridional (dashed) excursions from CM by drifter 72054, with $12 \mathrm{~h}$ low pass (solid) superimposed. By definition, excursions (and velocities) of drifter 72061 about the $\mathrm{CM}$ are $180^{\circ}$ out of phase with those of drifter 72054. Inset at bottom left shows zonal (solid) and meridional (dashed) velocity of drifter 72054 about the CM from the low-passed time series in the top right inset.

separated as one began a more rapid orbit around the south side of a warm-core eddy. Within 2 days, their separation distance had reached $27 \mathrm{~km}$. The behavior of this pair suggests that the drifters were trapped in a cyclonic submesoscale vortex with a core radius $\sim 3 \mathrm{~km}$ (far too small to be resolved in satellite altimetry, which resolves a minimum wavelength of $\sim 100 \mathrm{~km}$ [Fu and Ferrari, 2008]), rotating with a period close to the local inertial period of 0.83 days, i.e., Rossby number close to unity. This vortex may have been an example of a "spiral eddy" [Munk et al., 2000], a

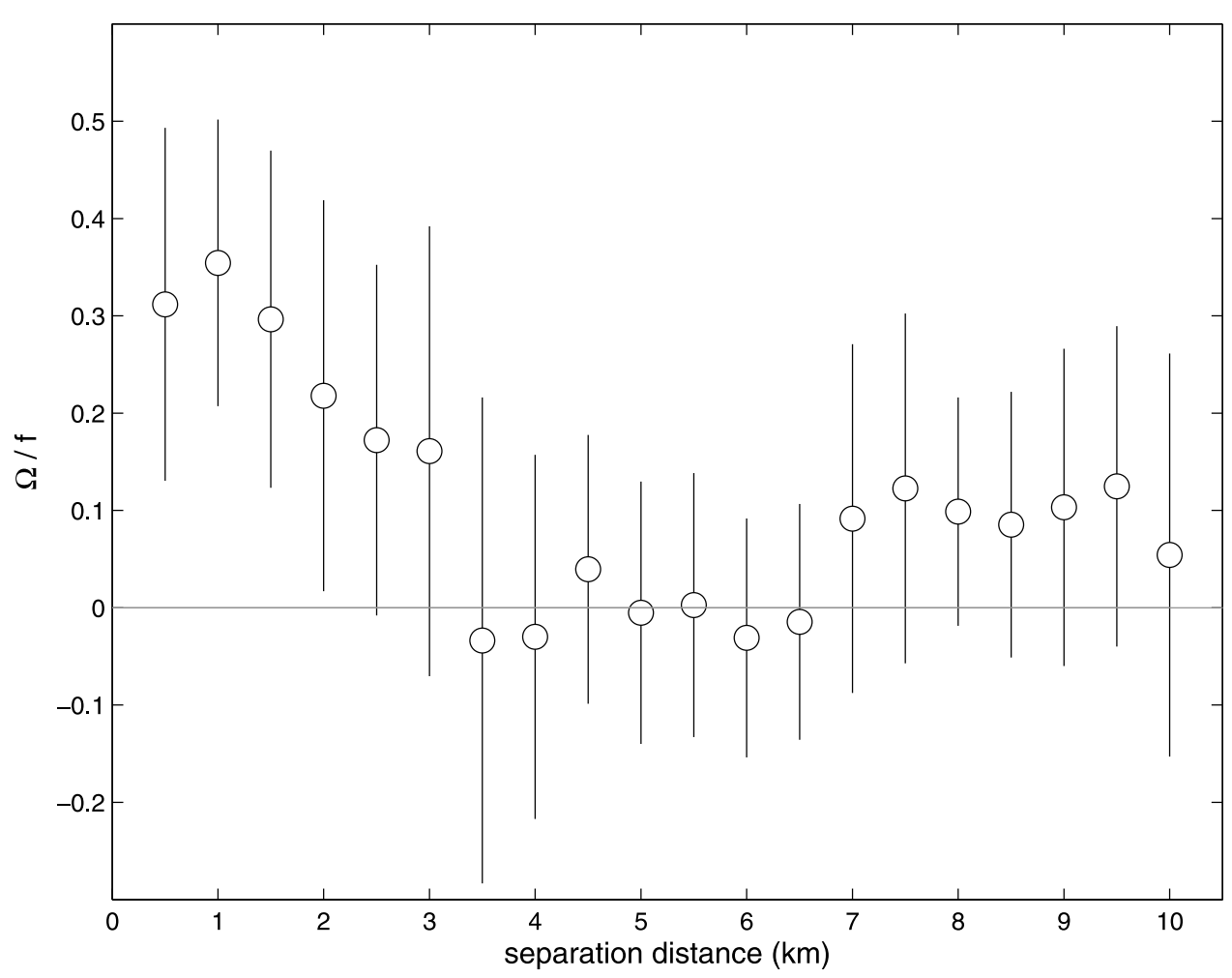

Figure 7. Lagrangian spin $\Omega$ nondimensionalized by Coriolis parameter $f$ of all CLIMODE drifter pairs about their center of mass, as a function of pair separation distance. Positive values are cyclonic. Standard error bars derived from standard deviation of all pairs in a $1 \mathrm{~km}$ bin and the number of $\Omega$ values in that bin, assuming that each day of measurements provides an independent observation. 


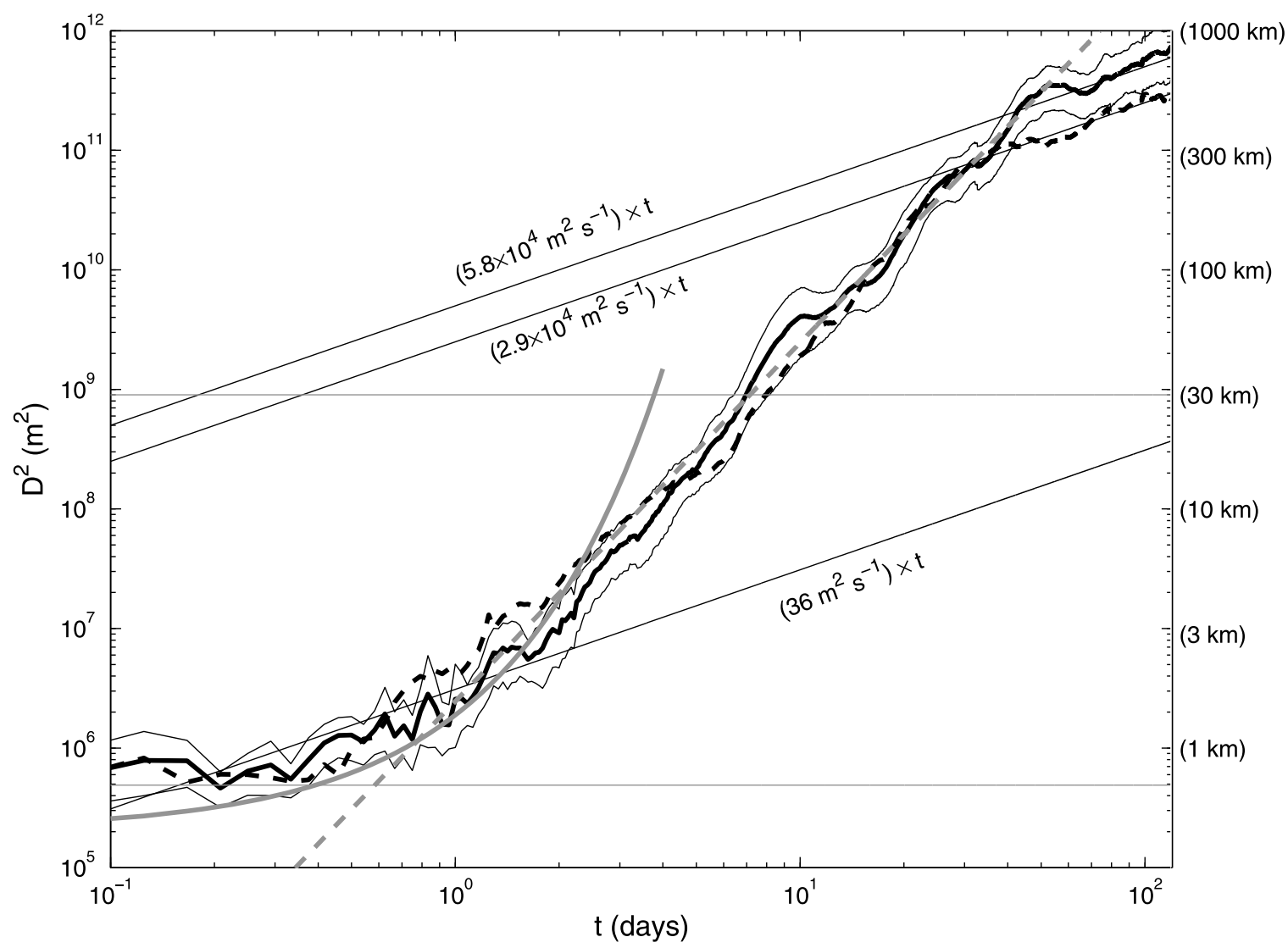

Figure 8. Zonal (heavy black solid curve) and meridional (heavy black solid dashed) dispersion of 55 CLIMODE drifter pairs. Axis on right indicates root-mean-square separation distance. Time $t$ is days after release. The $95 \%$ confidence intervals from bootstrap resampling are shown for zonal dispersion (thin black curves); errors in meridional dispersion are similar. Thin horizontal gray lines indicate the estimated noise level for separation, $(700 \mathrm{~m})^{2}$, and the squared deformation radius $(30 \mathrm{~km})^{2}$. Slanted black lines indicate random walk behavior with constant relative diffusivity $\left(\right.$ in $\mathrm{m}^{2} \mathrm{~s}^{-1}$ ) indicated by the labels. Best fits obeying Richardson's law in the range 1-40 days (gray dashed line) and exponential growth in the range $7 \mathrm{~h}$ to 1 day (heavy gray curve) are superimposed (see text).

predominantly cyclonic submesoscale feature observed in sun glitter and synthetic aperture radar imagery. These submesoscale vortices are believed to be formed by frontal baroclinic instability [Eldevik and Dysthe, 2002] or by inertial instability which magnifies cyclonic shear while weakening anticyclonic shear for large Rossby number flow [Shen and Evans, 2002].

[38] This pair was exceptional in the amount of time spent close together and in the clear cyclonic rotation about their center of mass. This rotation would not have been apparent in a lone drifter's trajectory. Other pairs exhibited briefer periods of cyclonic rotation about their center of mass when in close proximity. This can be quantified for all pairs by the Lagrangian spin, $\Omega=\left\langle u^{\prime} d v^{\prime}-v^{\prime} d u^{\prime}\right\rangle /(2 \Delta t$ EKE) where EKE $=1 / 2\left\langle u^{\prime 2}+v^{\prime 2}\right\rangle$ [Griffa et al., 2008] and $u^{\prime}, v^{\prime}$ is the drifter's motion with respect to the pair's center of mass. Note that by construction this calculation neglects spin associated with the pair orbiting as a coherent unit around mesoscale features, and cannot be equated with singleparticle spin such as in the global census of Griffa et al. [2008]. The average spin can be calculated as a function of pair separation distance, in $1 \mathrm{~km}$ bins, for all the
CLIMODE drifter pairs. The result (Figure 7) shows significant positive (cyclonic in the Northern Hemisphere) spin for pairs separated by less than $2.5 \mathrm{~km}$. Position noise, which will average $\Omega=0$, has most likely reduced these values; regardless, this result indicates a preference for cyclonic vorticity in the turbulent field at scales of $\mathrm{O}(1 \mathrm{~km})$.

\subsection{Quantifying Drifter Pair Spreading}

[39] Relative dispersion for the 55 CLIMODE drifter pairs is shown in Figure 8 in $\log$-log format. Up to 40 days after release, when the pairs reach a root-mean-square (rms) separation of $\sim 300 \mathrm{~km}$, their relative dispersion in the zonal and meridional directions is isotropic. For rms separation greater than $300 \mathrm{~km}$, meridional dispersion increases linearly with time, indicating random walk behavior with relative diffusivity $K=2.9 \times 10^{4} \mathrm{~m}^{2} \mathrm{~s}^{-1}$. At zonal rms separation greater than $500 \mathrm{~km}$, the zonal dispersion increases linearly with an effective relative diffusivity $K=5.8 \times 10^{4} \mathrm{~m}^{2} \mathrm{~s}^{-1}$. These values are equivalent to a single-particle diffusivity of $\kappa=K / 2=1.4-2.9 \times 10^{4} \mathrm{~m}^{2} \mathrm{~s}^{-1}$, consistent with earlier diffusivity estimates in this region [Lumpkin et al., 2002; Zhurbas and Oh, 2004]. Because the Gulf Stream extension 


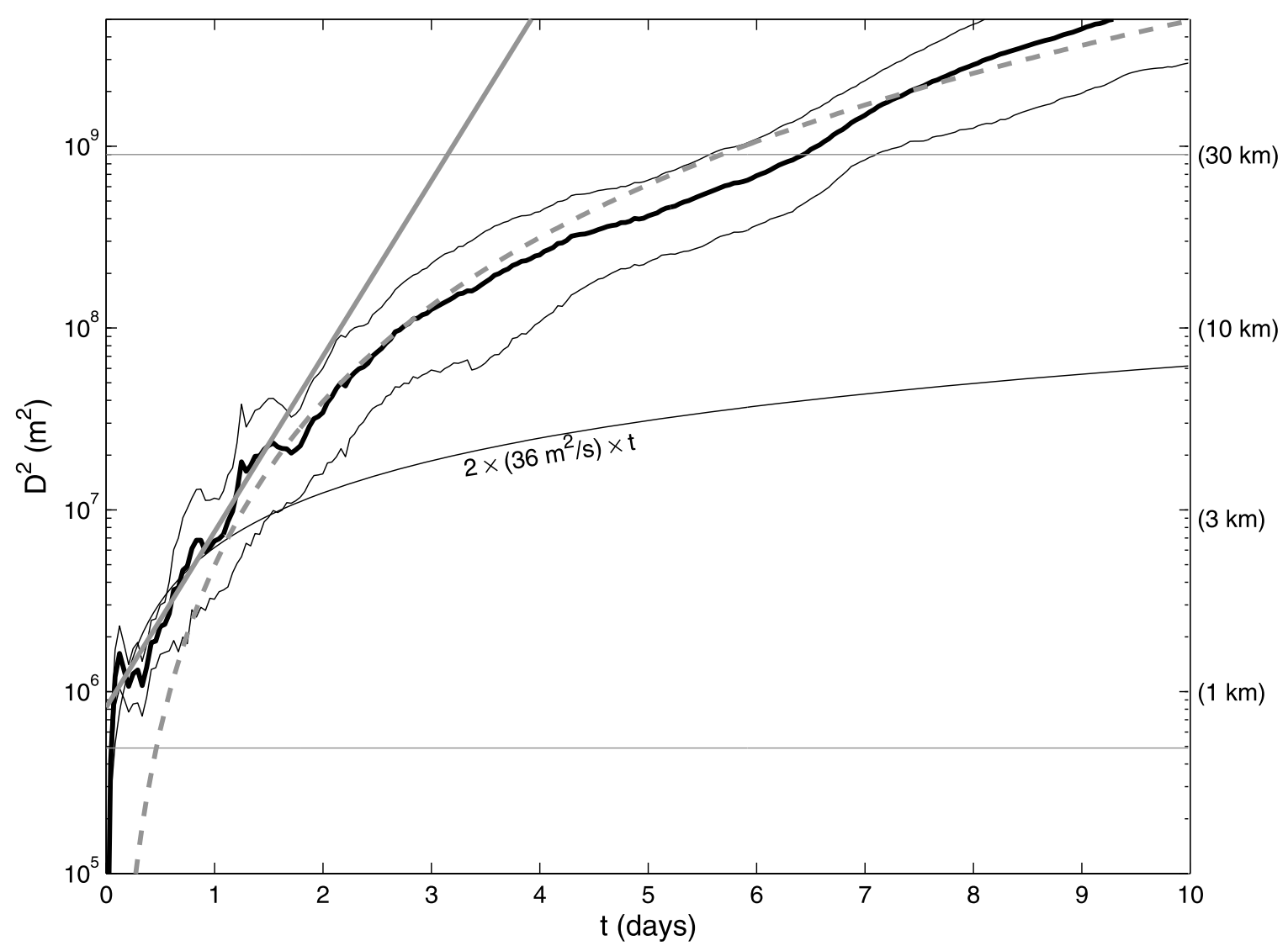

Figure 9. Mean squared separation distance (sum of zonal and meridional dispersion) of 55 CLIMODE drifter pairs, in semilog format (thick black curve), with $95 \%$ confidence intervals (thin black curves). Best fits of Richardson's law (thick gray dashed curve), exponential (thick gray solid curve), and diffusive behavior (thin black curve) are repeated from Figure 8, multiplied by a factor of 2 (assuming isotropic dispersion).

axis is effectively zonal at these scales, one can anticipate shear dispersion to magnify zonal diffusivity. Thus, the smaller meridional diffusivity may be most appropriate for characterizing the turbulent field at these scales [Zhurbas and $\mathrm{Oh}, 2004]$, with the transition to diffusive behavior at $300 \mathrm{~km}$ indicating the scale of the largest meanders and rings stirring the ocean surface.

[40] Of greater interest is the relative dispersion from the smallest resolvable scales $(\sim 700 \mathrm{~m})$ to the $300-500 \mathrm{~km}$ transition to diffusive behavior. The relative dispersion exceeds the noise level $(700 \mathrm{~m})^{2}$ at $7 \mathrm{~h}$ after release. A change in the slope of the dispersion curve at $\sim 1$ day after release (rms separation $2.6 \mathrm{~km}$ ), visible in $\log -\log$ (Figure 8) and semilog (Figure 9) formats, indicates a change in the dispersion regime. The early dispersion, from $7 \mathrm{~h}$ to 1 day, has a slope close to unity in log-log format, suggesting diffusive behavior, but also appears to be a nearly straight line in semilog format suggesting exponential growth. The least squares fit of a diffusive curve to the observed zonal/ meridional dispersion between $7 \mathrm{~h}$ and 1 day is $D_{\text {fit }}^{2}=$ $\left(36 \mathrm{~m}^{2} \mathrm{~s}^{-1}\right) t$. This fit exceeds the $95 \%$ confidence interval for the first $6 \mathrm{~h}$, and has an rms error of $730 \mathrm{~m}$. The best fit of an exponential curve in the same range is $D_{\text {fit }}^{2}=$ $\left[(640 \mathrm{~m}) \exp \left(1.08 \mathrm{~d}^{-1} t\right)\right]^{2}$, which briefly exceeds the $95 \%$ confidence interval for the observed $D^{2}$ at $7.5-9 \mathrm{~h}$ after release and has an rms error of $680 \mathrm{~m}$. It is interesting, albeit speculative, to note that an effective relative diffusivity of $K \sim 30-40 \mathrm{~m}^{2} \mathrm{~s}^{-1}$, consistent with our diffusive fit to $D^{2}$, is approximately what one would obtain from stirring length arguments $K \sim u L$ applied to waves and swell $\left(u \sim 1-2 \mathrm{~m} \mathrm{~s}^{-1}, L \sim 10-30 \mathrm{~m}\right)$. Wave conditions during the February-March 2007 CLIMODE cruise were extreme, with breaking seas driven by $20-25 \mathrm{~m} / \mathrm{s}$ winds common. It is possible that the drifter pairs were knocked about and randomly driven apart by wave-driven Stokes drift or other small-scale phenomenon until their rms separation reached $1-3 \mathrm{~km}$, when processes driving Richardson's law dispersion took over the spreading.

[41] Exponential separation cannot explain the behavior of the pairs at scales from $1-3 \mathrm{~km}$ to the first baroclinic Rossby radius of $\sim 30 \mathrm{~km}$. Instead, the observed dispersion clearly follows a $D^{2} \propto t^{3}$ relationship (Richardson's law) from 1 day after release (rms separation $1-3 \mathrm{~km}$ ) to the 40 day, $300 \mathrm{~km}$ transition to diffusive behavior (Figure 8). The least squares fit to the observed zonal/meridional dispersion in this range is $D_{\text {fit }}^{2}=\left(3.80 \times 10^{-9} \mathrm{~m}^{2} \mathrm{~s}^{-3}\right) t^{3}$.

[42] While it is traditional to present dispersion in zonal and meridional directions, it is also valuable to examine dispersion in a coordinate system that follows the meandering Gulf Stream front and associated large-scale features. 
(a)

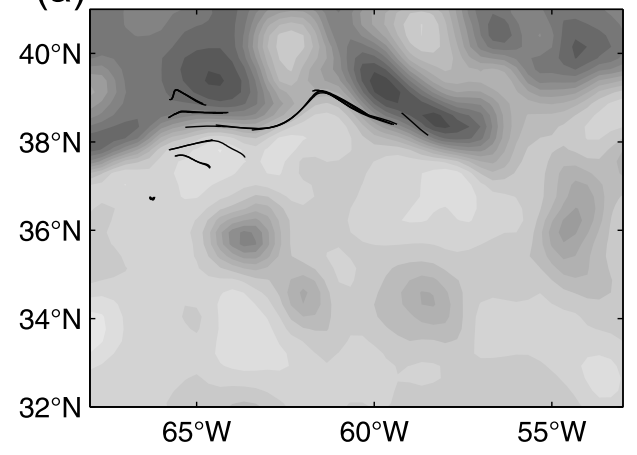

(b)

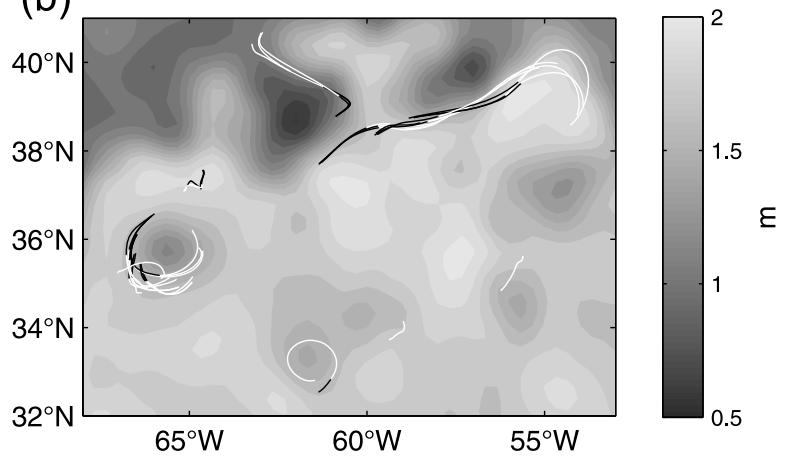

(c) $10^{12}$

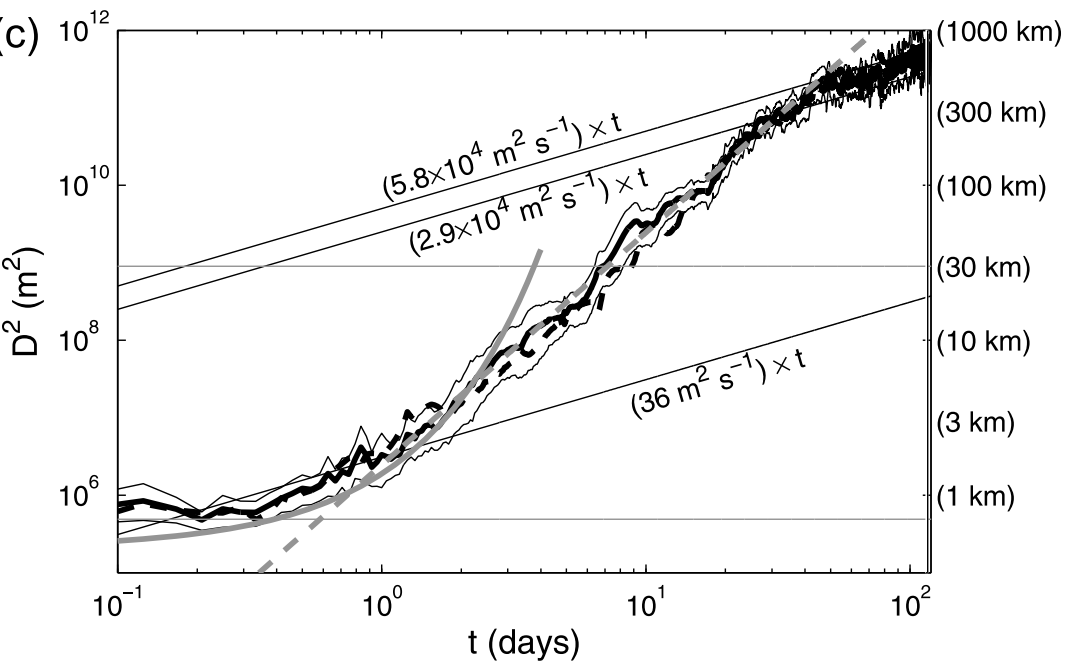

Figure 10. Absolute sea height (m) on (a) 14 February 2007 and (b) 14 March 2007. Superimposed trajectories are the paths of the drifter pairs' center of mass, for the week centered on the respective dates. The paths are black if they are during the first 8 days after release, and white for days 8-16. These paths are used to define a downstream and cross-stream (left of downstream) coordinate system. (c) Downstream (heavy black solid curve) and cross-stream (heavy black dashed curve) dispersion of CLIMODE drifter pairs. The 95\% confidence intervals are shown for downstream dispersion (thin black curves); errors in cross-stream dispersion are similar. Noise level, deformation radius, and best-fit lines copied from Figure 8.

We define a stream coordinate system using the center of mass of each of the drifter pairs, low passed at 2 days to remove inertial oscillations, fine-scale features, and noise in position fixes (Figures 10a and 10b). "Downstream" is defined as the direction taken by the low-passed center of mass, while "cross stream" is defined as $90^{\circ}$ to the left of downstream. The absolute velocities of the drifters become decorrelated after 8 days on average due to small-scale features in the turbulent field, but the low-passed center of mass continues to follow the geostrophic field to lowest order for many more days. Relative dispersion in this coordinate system is shown in Figure 10c. The pairs do not exhibit a significant different in downstream versus crossstream dispersion, arguing against the presence of shear dispersion which would magnify downstream dispersion relative to cross stream.

[43] Figure 11 shows the finite-scale Lyapunov exponents calculated from all possible CLIMODE drifter pairs, with $\alpha=1.15$ (see Appendix A). The FSLE curve is relatively flat at scales smaller than $2 \mathrm{~km}$, possibly indicating exponential separation, although this could also be caused by the hourly temporal resolution of the data, as the rare rapidly separating drifter pairs can reach $\mathrm{O}(2 \mathrm{~km})$ separation in an hour (this effect is explored in section 5 by resampling the data to daily resolution). In the scale range $2-10 \mathrm{~km}$, the FSLEs have a slope of -1 , consistent with $D^{2} \propto t^{2}$. This is slower than suggested by the relative dispersion (Figure 8), which has a $D^{2} \propto t^{3}$ slope for rms separations of $3-300 \mathrm{~km}$. The FSLE curve switches to a slope of -0.6 over the range $10-250 \mathrm{~km}$, close to the $-2 / 3$ slope expected for Richardson's law. At larger separations, the FSLE slope is close to -1.3 , indicating spreading at a rate between ballistic (slope -1 ) and diffusive (slope -2 ) dispersion.

\section{Discussion}

[44] The relative dispersion of the CLIMODE drifter pairs indicate that their separation grows according to Richardson's law for root-mean-square separation distances 


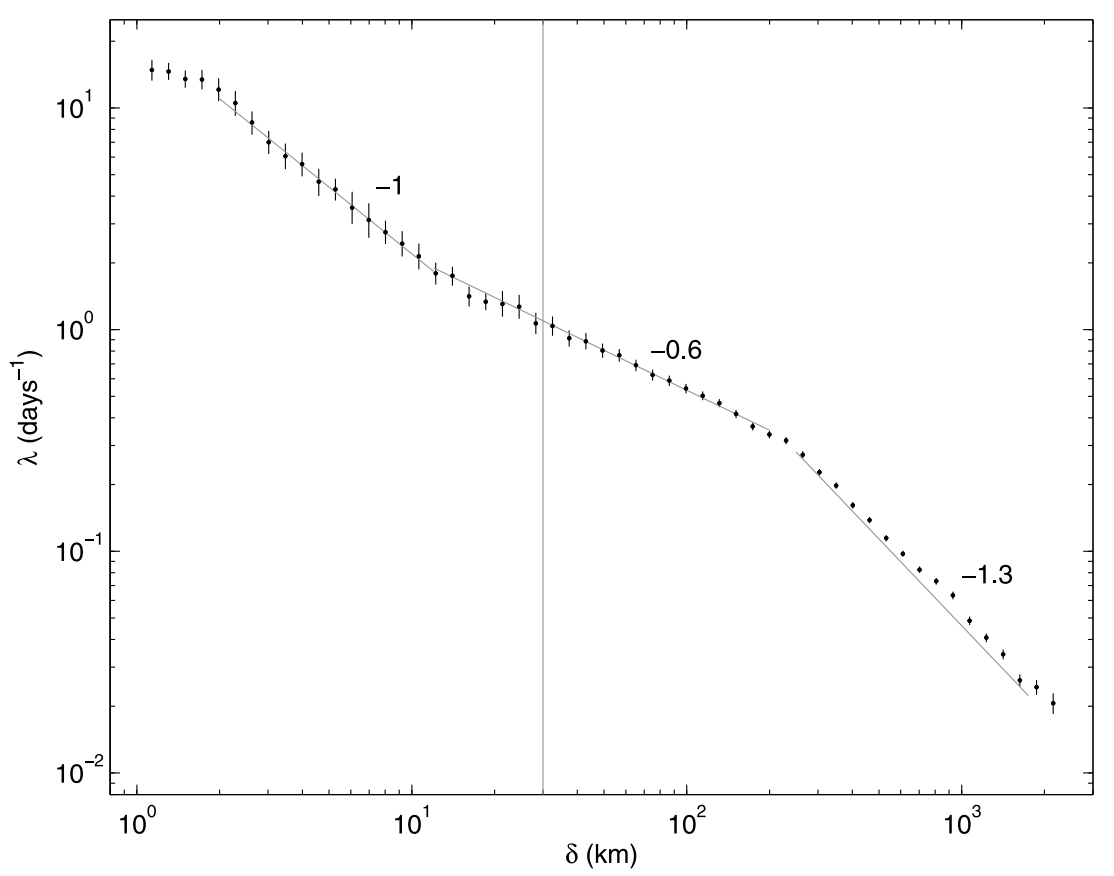

Figure 11. Finite-scale Lyapunov exponents calculated from all possible CLIMODE drifter pairs. The local first baroclinic Rossby radius is indicated by a vertical gray line.

from $2-3 \mathrm{~km}$ to $\sim 300 \mathrm{~km}$. The FSLE curve indicates a transition from slower spreading in the range $2-10 \mathrm{~km}$, to Richardson's law spreading at $10-250 \mathrm{~km}$. The local first baroclinic Rossby radius is $\sim 30 \mathrm{~km}$ [Chelton et al., 1998]. Both the relative dispersion and the FSLEs indicate that the separation from $1-3 \mathrm{~km}$ to the Rossby radius is not exponential.

[45] Our results are qualitatively consistent with earlier float studies in the Gulf Stream and North Atlantic Current region. LaCasce and Bower [2000] found relative dispersion and FSLEs consistent with Richardson's law behavior for $700 \mathrm{~m}$ deep isobaric floats in the Site L, North Atlantic Current and Local Dynamics experiments, at separations of $10 \mathrm{~km}$ to $100-200 \mathrm{~km}$. This behavior is very different from what has been found in the eastern North Atlantic, where Ollitrault et al. [2005] observed clear exponential separation at scales smaller than the Rossby radius for 16 float pairs at 700 dbar (their results at submesoscale for 9 float pairs in the western Atlantic were not robust, due to the small number of pairs). LaCasce and Bower [2000] also examined float pairs in the eastern North Atlantic, and found dispersion weaker than Richardson's, but also noted that the separation at submesoscale was not well resolved with their data. At scales up to the Rossby radius, our results contrast with the Gulf of Mexico drifter study of LaCasce and Ohlmann [2003] and the Nordic Seas study of Koszalka et al. [2009], both of whom found exponential separation while we find Richardon's law separation.

[46] Why does pair spreading at scales from $\sim 2 \mathrm{~km}$ to the local Rossby radius follow Richardson's law in some studies, and is exponential in others? One hypothesis worth briefly considering is the fact that many studies have relied upon chance pairs, which may bias observations toward regions where the surface field is convergent. Although earlier balloon dispersion studies [Morel and Larcheveque, 1974; Er-el and Peskin, 1981] did not find a statistical difference between chance and original pairs, LaCasce and Ohlmann [2003] speculated that this might affect their results but could not quantify the effect with their data set. However, this hypothesis seems unlikely to explain the disparate results of the float and drifter studies in the Atlantic at the submesoscale: both this study and Ollitrault et al. [2005] rely on original pairs, with Richardson's separation found here and Ollitrault et al. [2005] finding exponential separation. LaCasce and Bower [2000] and LaCasce and Ohlmann [2003] relied on chance pairs and found Richardon's law and exponential separation, respectively.

[47] We can test the impact of chance pairs versus original pairs in the Gulf Stream region by calculating the dispersion and FSLEs for the chance drifter pairs in the region. The results (Figure 12) have large error bars for relative dispersion, because of the small number of chance drifter pairs with initial separation distance of $700 \mathrm{~m}$ or less (nine pairs). Within these large error bars, the relative dispersion can accommodate an exponential growth or a $D^{2} \propto t^{3}$ Richardson's law growth at scales from $1-3 \mathrm{~km}$ to the local Rossby radius. However, only the Richardson's law curve fits a broader range of scales, out to the $\sim 300 \mathrm{~km}$ transition to diffusive behavior. The FSLEs (calculated with $\alpha=1.5$ ) have nearly the same slopes as for the CLIMODE drifters (Figure 11), although they tend to be slightly smaller in magnitude. Because the relative dispersion and FSLEs for the chance pair drifters are nearly identical to those of the CLIMODE drifters within the confidence intervals, we conclude that these calculations are not contaminated by the inhomogeneous sampling of chance pairs, at least in the Gulf Stream region. 
(a)

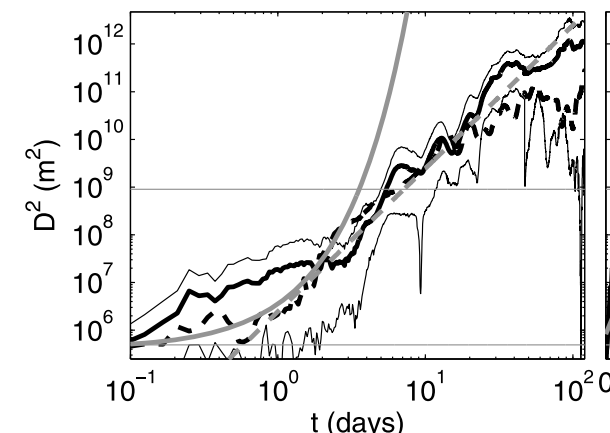

(b)

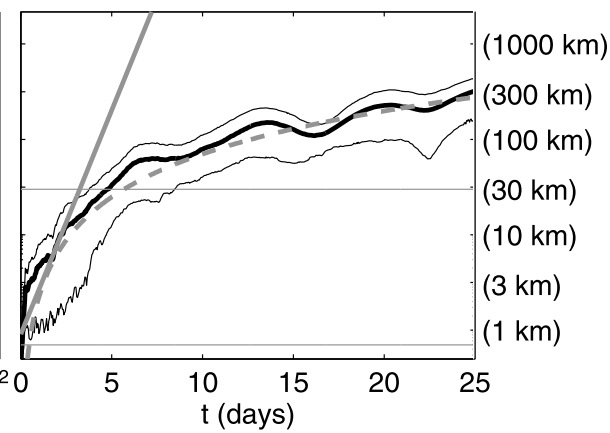

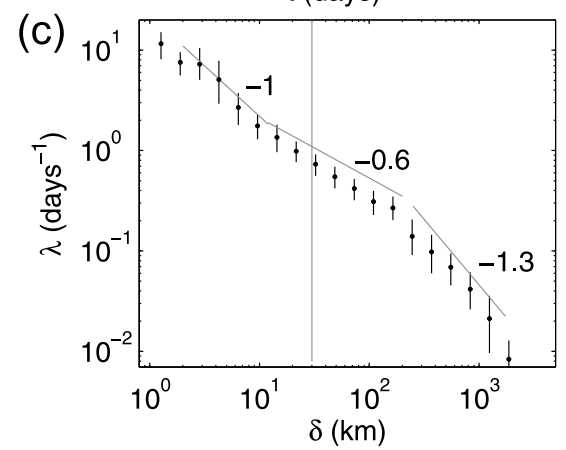

Figure 12. (a) Zonal (solid black curve) and meridional (dashed black curve) dispersion for the nine chance pairs in the Gulf Stream region with initial separation distance $<700 \mathrm{~m}$. The $95 \%$ error bars for zonal dispersion shown by thin lines. Exponential (heavy solid gray curve) and Richardson's law (heavy dashed gray curve) fits to the CLIMODE drifters (Figure 8) are superimposed for reference. Horizontal gray lines indicate the squared noise level for pair separation distance (lower) and the squared local Rossby radius of deformation (upper). (b) Squared distance (sum of zonal and meridional dispersion) versus time, in semilog format. Gray curves repeated from Figure 12a, assuming isotropic dispersion. (c) Finite-scale Lyapunov exponents, with $95 \%$ confidence intervals. The local first baroclinic Rossby radius is indicated by a vertical gray line; the other gray lines indicate values and slopes of the CLIMODE drifter pairs (Figure 11) for reference.

[48] A second hypothesis for the discrepancy in the submesoscale dispersion from the various drifter and float studies is that regional differences in dynamics are associated with qualitatively different dispersion regimes at the submesoscale. For example, the Gulf of Mexico drifters of LaCasce and Ohlmann [2003] were in a region of relatively weak surface fronts north of the Loop Current and in the far northwestern Gulf. The CLIMODE drifters, in contrast, were on or near the extremely energetic Gulf Stream front in the presence of intense wind forcing, conditions that can lead to frontogenesis of submesoscale vortices [Thomas et al., 2008]. As noted earlier, pair dispersion following Richardson's law at separation greater than 1-3 km indicates the presence of energetic eddies at this scale, far smaller than the local first baroclinic Rossby radius of $\sim 30 \mathrm{~km}$. Submesoscale coherent vortices [McWilliams, 1985] of $\mathrm{O}(1 \mathrm{~km})$ radius were directly observed in SeaSoar measurements during the CLIMODE cruise ( $T$. Joyce and L. Thomas, personal communication, 2007), and in a few drifter trajectories (e.g., Figure 6). Perhaps the presence of energetic features at the scales of these vortices in the Gulf Stream region, and and their absence in more quiescent parts of the ocean, could account for the discrepancy between our results and those of LaCasce and Ohlmann [2003]. This result is consistent with the modeling studies of Isern-Fontanet et al. [2006] and Lapeyre [2009], who found that the surface mode of SQG theory (with its relatively flat wave number spectrum) dominates the flow in regions with large-mesoscale energy and in regions with deep mixed layers. In contrast, the interior baroclinic mode (described by QG theory) dominates the surface in less energetic regions such as the Gulf of Mexico, although those studies did not extend to the Gulf. It is interesting to note, however, that the $700 \mathrm{~m}$ deep floats in the western North Atlantic examined by LaCasce and Bower [2000] also demonstrated Richardson's law dispersion at scales smaller than the local Rossby radius. This is deeper than the surface-trapped mode of SQG, and demands an alternative explanation.

[49] We test the hypothesis of regional dependence with the ADB drifters deployed in the eastern subtropical Atlantic. We do not argue that the submesoscale energy in the eastern subtropical North Atlantic is comparable to that of the northern and western Gulf of Mexico, but merely that these regions may be qualitatively different from the Gulf Stream front.

[50] The relative dispersion and FSLE curves for the ADB drifters are shown in Figure 13. The dispersion is slower than observed in the CLIMODE array, and the local Rossby deformation radius is larger $(40 \mathrm{~km})$ [Chelton et al., 1998]. 
(a)

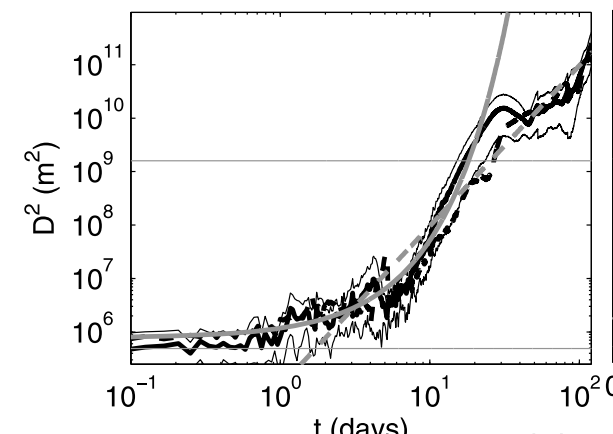

(b)

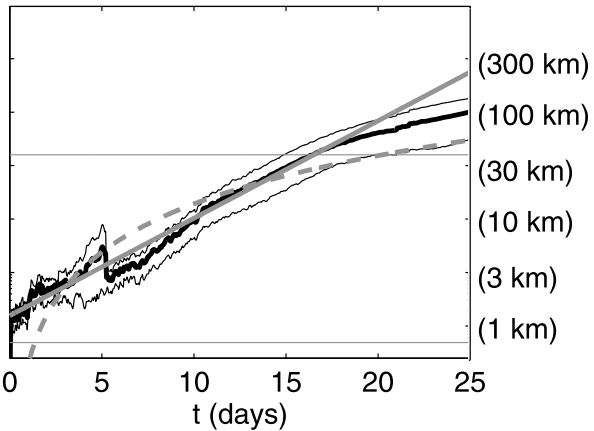

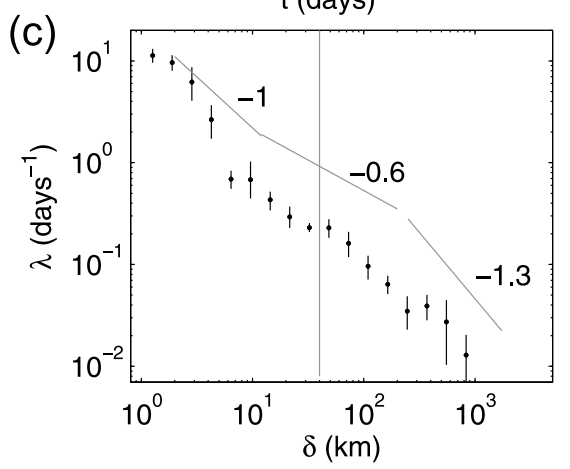

Figure 13. (a) Zonal (solid black curve) and meridional (dashed black curve) dispersion for the $18 \mathrm{ADB}$ drifter pairs in the eastern subtropical Atlantic with initial separation distance $<700 \mathrm{~m}$. The 95\% error bars for zonal dispersion shown by thin lines. Exponential (solid gray curve) and Richardson's law (dashed gray curve) fits to the behavior in the range 1-15 days are superimposed (see text). Horizontal gray lines indicate the squared noise level for pair separation distance (lower) and the squared local Rossby radius of deformation (upper). (b) Squared distance (sum of zonal and meridional dispersion) versus time, in semilog format. Gray curves repeated from Figure 13a, assuming isotropic dispersion. (c) Finite-scale Lyapunov exponents, with $95 \%$ confidence intervals. The local first baroclinic Rossby radius is indicated by a vertical gray line; the other gray lines indicate values and slopes of the CLIMODE drifter pairs (Figure 11) for reference.

As a consequence the pairs required an average of 15 days to reach a root-mean-square separation distance equal to the deformation radius, versus 7 days for the CLIMODE drifters. At lags greater than $\sim 30$ days, the dispersion in both zonal and meridional directions grows approximately linearly with time, consistent with a single-particle diffusion of $\kappa=1.7 \times 10^{3} \mathrm{~m}^{2} \mathrm{~s}^{-1}$, nearly an order of magnitude lower than in the CLIMODE region. A discontinuity in the dispersion occurs at 4-6 days after release, when several of the ADB drifters failed and the data abruptly became more sparse (from 18 pairs to 15 pairs). A least squares fit of a Richardson's law curve to the observed zonal/meridional dispersion in the range $1-15$ days (Figure 13) is $D_{\text {fit }}^{2}=(1.49 \times$ $\left.10^{-10} \mathrm{~m}^{2} \mathrm{~s}^{-3}\right) \times t^{3}$. This fit exceeds the $95 \%$ confidence estimate of $D^{2}$ between the discontinuity at 5 and 10 days, and below the confidence interval on days 1-2.5. It has an rms error of $5.7 \mathrm{~km}$. A best fit of an exponential in the $1-15$ day range is $D_{\text {fit }}^{2}=\left[(880 \mathrm{~m}) \exp \left(0.21 \mathrm{~d}^{-1} t\right)\right]^{2}$. This fit exceeds the confidence interval from 5 days to 8.5 days, and has an rms error of $4.0 \mathrm{~km}$. Given the large error bars on the observed dispersion, it is difficult to conclusively discriminate between Richardson's law and exponential dispersive behavior in this range, although exponential growth fits the observations (Figure 13) better.
[51] The FSLEs for the ADB drifters (Figure 13c), calculated using $\alpha=1.5$, are close to the CLIMODE results at scales smaller than a few kilometers, but drop off more quickly in the range $3-5 \mathrm{~km}$ with a slope of -2 to -3 . Between 4 and $6 \mathrm{~km}$, the FSLEs switch to a $-2 / 3$ slope that persists to at least $350 \mathrm{~km}$ separation. Beyond $350 \mathrm{~km}$, the FSLEs may drop off more quickly again, consistent with diffusive behavior, but this is not robust. The $-2 / 3$ slope in the FSLEs indicates Richardson's law dispersion in this scale range, inconsistent with exponential separation (constant FSLEs). More observations are available for calculating the FSLEs than for relative dispersion from an initial separation distance $<700 \mathrm{~m}$. For example, at 10 days lag, when the rms separation of the ADB pairs was $10 \mathrm{~km}$, 14 pairs remained for calculating relative dispersion. For the FSLE calculation, there were 31 cases where ADB pairs separated from $9.9 \mathrm{~km}$ to $11.4 \mathrm{~km}$ (the $\left[\delta_{n}, \delta_{n+1}\right]$ bin bracketing $10 \mathrm{~km}$ ). Thus, in the eastern subtropical Atlantic, the relative dispersion suggests exponential spreading at scales smaller than the Rossby radius, while the FSLE results indicate that it is not exponential at scales from $\mathrm{O}(1 \mathrm{~km})$ to the deformation radius. Obviously, any conclusions must be more tentative for the ADB drifters 
(a)

(b)
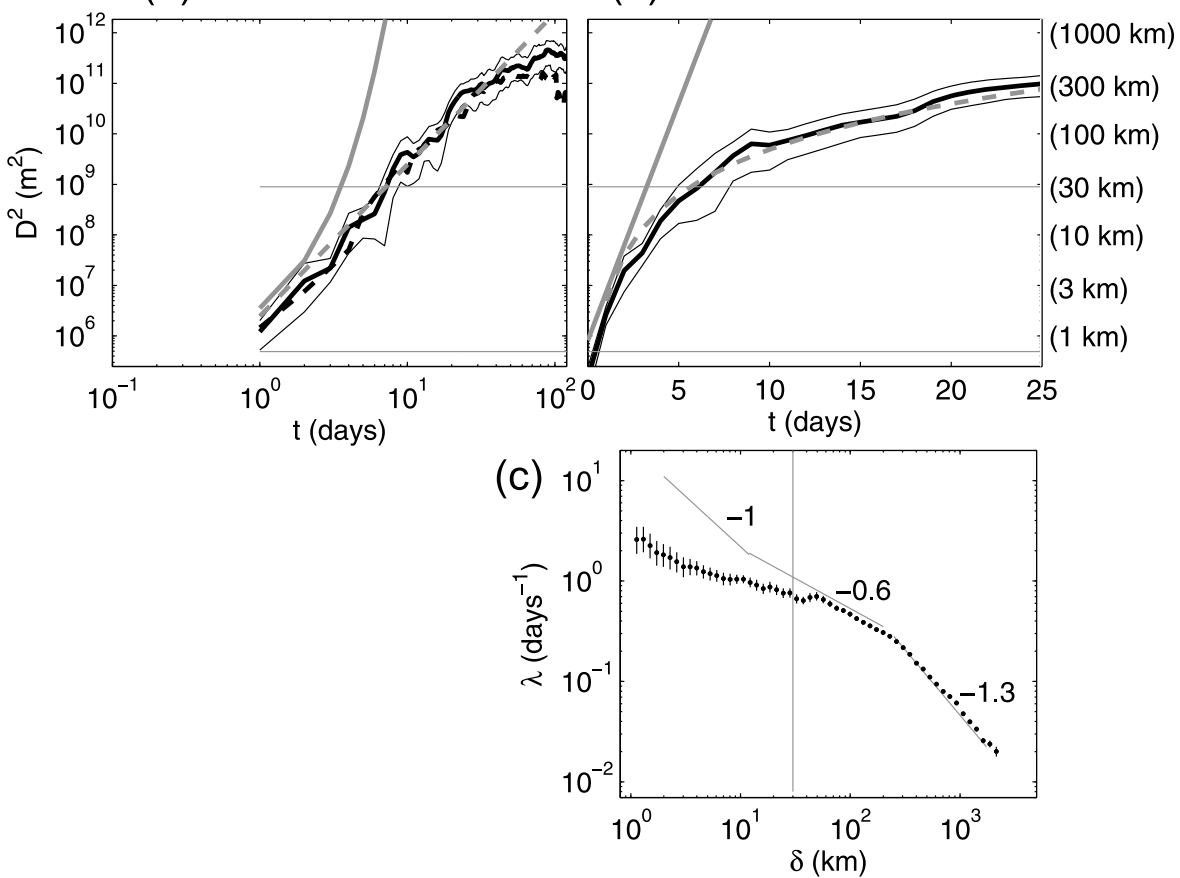

Figure 14. (a) Zonal (solid black curve) and meridional (dashed black curve) dispersion for the CLIMODE drifter pairs, subsampled to daily resolution. The $95 \%$ error bars for zonal dispersion shown by thin lines. Exponential (solid gray curve) and Richardson's law (dashed gray curve) fits from the hourly resolution data (Figure 8) are superimposed. Horizontal gray lines indicate the squared noise level for pair separation distance (lower) and the squared local Rossby radius of deformation (upper). (b) Squared distance (sum of zonal and meridional dispersion) versus time, in semilog format. Gray curves repeated from Figure 14a, assuming isotropic dispersion. (c) Finite-scale Lyapunov exponents, with 95\% confidence intervals. The local first baroclinic Rossby radius is indicated by a vertical gray line; the other gray lines indicate values and slopes of the hourly resolution CLIMODE drifter pairs (Figure 11) for reference.

than for the much denser CLIMODE data set, where the relative dispersion and FSLE results were consistent.

[52] It is also worth noting that the FSLE results of LaCasce and Ohlmann [2003] may have been contaminated by the temporal resolution of the data [LaCasce, 2008], because rapidly separating pairs were not sufficiently resolved. In 2003, the Argos network provided a maximum of 6-8 fixes per day, and periods with only a few fixes per day were not uncommon [Elipot and Lumpkin, 2008]. We can examine this effect on our data by subsampling the CLIMODE data to daily resolution and recalculating the pair spreading. The results are shown in Figure 14: subsampling alters the resolution of the relative dispersion curves, but not their slopes. However, subsampling has a profound effect on the FSLE results: the curve is flattened significantly [LaCasce, 2008] at separation distances smaller than $50 \mathrm{~km}$. The slope doesn't become perfectly flat at these scales, but the slope of -0.35 to -0.4 spuriously indicates dispersion far faster than the fastest "normal" power law dispersion (Richardson's law, with an FSLE slope of $-2 / 3$ ).

[53] These results from the subsampled CLIMODE data suggest that the FSLE values of LaCasce and Ohlmann [2003] were contaminated by the temporal resolution of those data at scales smaller than a threshold $\delta_{\min }$. If the temporal resolution of the data is $\mathrm{d} t$, there will be a small but significant fraction of pairs that separate at a speed $v_{\max }$ which sets $\delta_{\min }=\mathrm{d} t \cdot v_{\max }$. Our results for the subsampled CLIMODE drifters indicate that $v_{\max }=(50 \mathrm{~km}) /$ (1day) $\sim 50 \mathrm{~cm} \mathrm{~s}^{-1}$. As noted earlier, there were a small number of cases $(<1 \%)$ where CLIMODE pairs separated at this speed. This implies that our FSLE curve for fullresolution (hourly) data is suspect at scales smaller than $\sim 2 \mathrm{~km}$.

\section{Conclusions}

[54] In this study, we have examined the relative dispersion and finite-scale Lyapunov exponents of drifter pairs in the CLIMODE array, and some other recent drifter pairs in the North Atlantic. The CLIMODE drifters and chance drifter pairs in the Gulf Stream region exhibit Richardson's law relative dispersion from $1-3 \mathrm{~km}$ to $300-500 \mathrm{~km}$, the scale of the largest rings and meanders, beyond which their dispersion is consistent with a single-particle constant effective diffusivity of $\mathrm{O}\left(1-2 \times 10^{4} \mathrm{~m}^{2} \mathrm{~s}^{-1}\right)$. Drifters in the eastern subtropical North Atlantic spread more slowly. FSLEs in this region indicate Richardson's law spreading at subdeformation length scales, but the relative dispersion is more consistent with exponential spreading, yet are more noisy. The long-term effective diffusivity in this region is smaller by an order of magnitude than in the Gulf Stream. 
[55] For separation scales larger than the local deformation radius our results are consistent with numerous previous drifter and float studies, indicating an energy cascade up to the scale of the largest eddies, then a transition at larger separations to random walk behavior characterized by a constant effective diffusivity.

[56] In the scale range from $1-3 \mathrm{~km}$ to the local deformation radius of $\sim 30 \mathrm{~km}$, our results indicate that the velocity wave number spectrum in the Gulf Stream region is flatter than the $k^{-3}$ slope predicted for the enstrophy cascade subrange of 2-D quasigeostrophic turbulence [Kraichnan, 1967; Charney, 1971]. A $k^{-3}$ slope would produce exponential pair separation [Bennett, 1984] and constant FSLEs. Our results indicate local (nonexponential) spreading in this scale range, consistent with a flatter wave number spectrum. Shear dispersion could produce Richardson's law-like relative dispersion in the direction parallel to the sheared flow [Bennett, 1987], but also produces weaker dispersion in the cross-flow direction. In contrast, we find isotropic spreading in the $2-30 \mathrm{~km}$ scale range, in both geographic and stream coordinates, inconsistent with shear dispersion.

[57] The presence of a rich and energetic submesoscale field has been documented in several recent high-resolution simulation studies [Klein et al., 2008; Capet et al., 2008], in which the energy of the submesoscale increases dramatically when the simulation resolution approaches $\mathrm{O}(1 \mathrm{~km})$. Submesoscale coherent vortices (SCVs) [see McWilliams, 1985] at the ocean surface are generated by frontal baroclinic instability [Eldevik and Dysthe, 2002] or inertial instability [Shen and Evans, 2002]. The presence of an energetic submesoscale field, with $\mathrm{O}(1)$ Rossby and Richardson numbers with vertical velocities an order of magnitude larger than the mesoscale field, has significant impact on the structure of the mixed layer [Thomas et al., 2008]. Observations [Munk et al., 2000; Rudnick, 2001; Griffa et al., 2008], simulations [Klein et al., 2008], and theory [Shen and Evans, 2002; Klein et al., 2003] suggest that SCVs at the ocean surface are predominantly cyclonic, a result consistent with the spin of the CLIMODE drifter pairs about their center of mass at separation distances smaller than $2.5 \mathrm{~km}$ (Figure 7).

[58] Surface quasigeostrophic (SQG) theory predicts a shallower wave number spectrum than in 2-D geostrophic turbulence at scales smaller than the Rossby radius, consistent with the Gulf Stream CLIMODE drifters. Because SQG assumes that the surface velocity field is completely driven by surface density variations, it predicts that sea height and surface density (or its measured proxy, SST) are strongly correlated and thus surface currents can be calculated from snapshots of SST [Isern-Fontanet et al., 2006] where SQG applies. Recently, Isern-Fontanet et al. [2008] exploited this relationship to calculate surface currents from SST in a fully eddy-resolving numerical simulation of the North Atlantic. They found that the SQG approach was able to accurately estimate the model's currents in regions where the vorticity was large (although not so large that nonlinear terms became important), and where SST variations followed density variations at the base of the mixed layer. This latter condition was met in regions with strong winds, and in regions with deep mixed layers including the Sargasso Sea where winds are relatively weak. They mapped the skill of the SQG approach between 32 and $48^{\circ} \mathrm{N}$ (their Figure 5), and demonstrated that it works well over much of the CLIMODE study region, except over the Grand Banks characterized by shallow mixed layers. Although their study did not extend to the Gulf of Mexico, their results and the generally shallower mixed layers of the Gulf suggest that SQG may not be as relevant for surface drifters in that region. In another study of an eddy-resolving simulation of the North Atlantic, Lapeyre [2009] found that the surface mode of SQG theory dominates the flow in regions of intense mesoscale activity (such as the CLIMODE study region) while the interior first baroclinic mode, with its associated steeper wave number spectrum at the submesoscale, dominates in relatively quiescent regions such as the northern Gulf of Mexico or the eastern subtropical North Atlantic.

[59] While SQG theory is fully consistent with the results of this study, there are some significant questions which remain regarding its ability to explain the full suite of earlier studies. It is not necessarily inconsistent with surface drifters in the Gulf of Mexico [LaCasce and Ohlmann, 2003] demonstrating exponential separation at submesoscales due to the regional differences discussed earlier. Similarly, it is not inconsistent with exponential dispersion of subsurface floats [Ollitrault et al., 2005] in the submesoscale range because those observations were beneath the $\mathrm{O}(250 \mathrm{~m})$ thick surface boundary layer and in the less energetic eastern North Atlantic. However, SQG cannot account for submesoscale Richardson's law-like dispersion of $700 \mathrm{~m}$ deep floats in the Gulf Stream region [LaCasce and Bower, 2000], which should be well beneath the influence of the surface-trapped mode. In addition, the Nordic Seas drifters of Koszalka et al. [2009] demonstrated exponential separation at scales smaller than the local Rossby radius, in a region of substantial eddy kinetic energy and deep mixed layers, although this exponential separation was observed only over the first 2 days after release; it is plausible that their $25 \mathrm{~h}$ window filtering and $6 \mathrm{~h}$ subsampling may have impacted the submesoscale energy in their observations. In a recent study of velocity and temperature spectra across the Gulf Stream measured by repeat ADCP sections from the Oleander Project, Wang et al. [2010] found a $k^{-3}$ spectral slope from $\sim 20 \mathrm{~km}$ to $\sim 200 \mathrm{~km}$ at depths of $30-90 \mathrm{~m}$. This result is consistent with the enstrophy cascade of 2-D QG turbulence, but should yield exponential separation of particle pairs across the full mesoscale range to the largest energy-containing rings and meanders. Clearly the stark contrast between the results of Wang et al. [2010] and this study is startling, and the relevance of SQG in the western North Atlantic remains subject to reevaluation in future analyses of observations.

[60] At separation distances less than $1 \mathrm{~km}$, pair spreading may be dominated by the larger submesoscale vortices, and in principle one would expect the relative dispersion to be nonlocal (exponential). The slope of the CLIMODE drifters' relative dispersion at rms separation from $800 \mathrm{~m}$ to $2 \mathrm{~km}$ is consistent with exponential separation (Figure 9), and we find a constant value for the FSLEs at these small separation distances. It is tempting to interpret these results as consistent with nonlocal spreading driven by coherent submesoscale vortices. However, as noted earlier, the relative dispersion can also be explained by a constant diffusivity in this range, and it is at these small scales that the hourly 


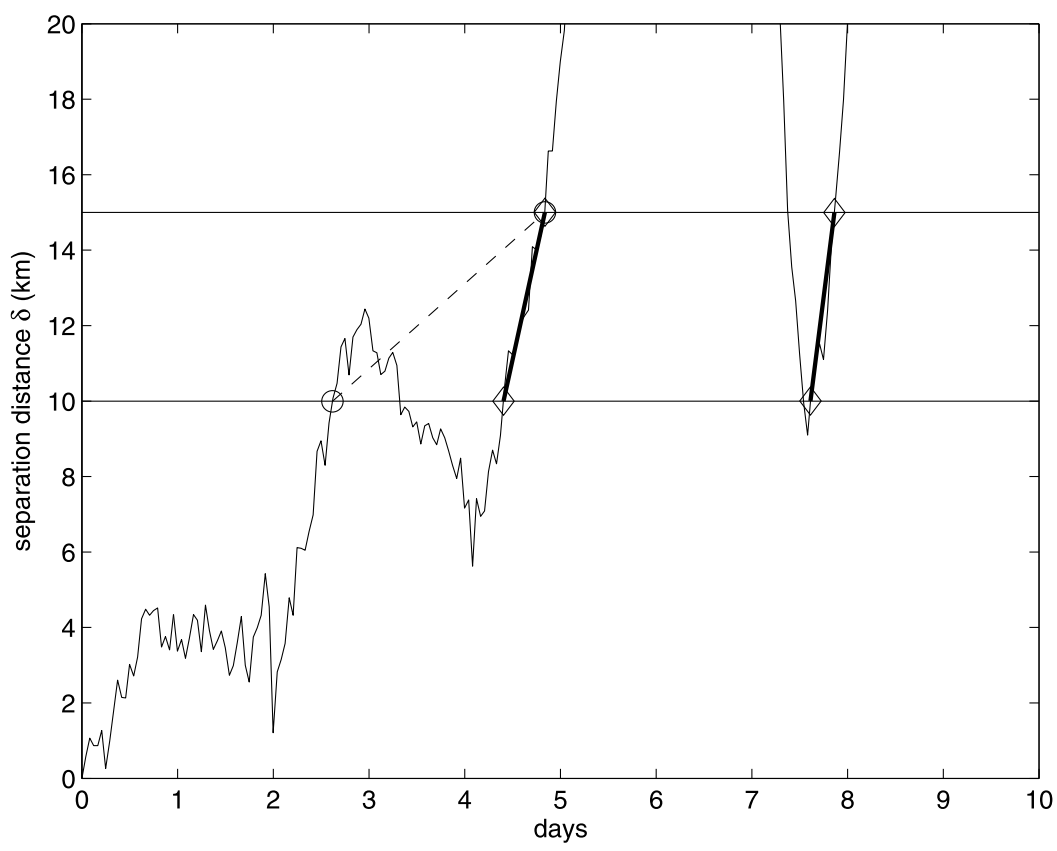

Figure A1. Plot of separation distance versus time since deployment for one of the CLIMODE drifter pairs. Suppose the FSLE $\lambda_{n}$ is calculated over the interval $\left[\delta_{n}=10 \mathrm{~km}, \delta_{n+1}=15 \mathrm{~km}\right]$. The first crossing method would identify the circled intersections connected by a dashed line, with an interval $\Delta t_{n}=2.22$ days. The fastest crossing method would identify two crossings indicated by the diamond intersections connected by heavy black lines, with intervals of 0.43 and 0.25 days.

resolution of our data may contaminate our FSLE results. Thus, higher temporal resolution data are necessary to evaluate pair spreading at scales below approximately $2 \mathrm{~km}$.

[61] Finally, it is important to note that this study's conclusions do not imply that tracer spreading is not exponential. In local dispersion, when the stirring is dominated by eddies of the same scale as a tracer cloud, the cloud becomes highly convoluted, the length of the cloud can be stretched exponentially, while its end points (and particle pair separation) follows Richardson's law [Bennett, 1984]. In contrast, in nonlocal dispersion, stirring is dominated by much larger eddies. The tracer cloud is then drawn into a few long streaks, and pair separation is exponential [Bennett, 1984]. Thus, there is no qualitative inconsistency between this study's results and recent studies indicating exponential stretching of the length of a tracer cloud at the ocean surface [Beron-Vera et al., 2008; Waugh and Abraham, 2008]. However, many recent studies such as these rely upon eddy velocity fields derived from the AVISO product [Le Traon et al., 1998], which does not contain significant energy at scales smaller than $\sim 200 \mathrm{~km}$. The apparently ubiquitous presence of an energetic submesoscale field at the ocean surface may elevate initial tracer spreading rates substantially above the rates calculated in those studies.

\section{Appendix A: Details of Calculating Finite-Scale Lyapunov Exponents}

[62] Finite-scale Lyapunov exponents can be sensitive to the temporal spacing of the data at small separation distances [LaCasce, 2008], for which the time interval $\Delta t$ approaches the resolution of the data for some pairs. In this study, we attempt to ameliorate this issue by calculating the times corresponding to $\delta_{n}$ via linear interpolation between the closest hourly values bracketing $\delta_{n}$ (A. Haza, personal communication, 2009). For example, if a pair goes from $\delta=1 \mathrm{~km}$ to $\delta=1.4 \mathrm{~km}$ in $1 \mathrm{~h}$ (the temporal resolution of our data), and we seek a $\Delta t$ for the transition from $\delta_{n}=1 \mathrm{~km}$ to $\delta_{n+1}=1.2 \mathrm{~km}$, we would derive $\Delta t=$ $0.5 \mathrm{~h}$. For the CLIMODE drifter pairs, the separation speed from the center of mass was less than $50 \mathrm{~cm} \mathrm{~s}^{-1}$ for $99.3 \%$ of the hourly observations; a rare $50 \mathrm{~cm} \mathrm{~s}^{-1}$ separation over $1 \mathrm{~h}$ translates to a spatial scale of $1.8 \mathrm{~km}$, so our FSLE values at smaller scales may be contaminated by the temporal resolution of the data. This temporal sampling limit affects not only the lowest value of $\lambda$ that can be resolved, but also the minimum value of $\alpha$ that can be chosen [Haza et al., 2008]. Lower values of $\alpha$ provide denser resolution of the FSLEs as a function of $\delta$, but below a data-dependent threshold the values and slopes of the FSLEs will be affected for small separations. In this study, we followed the approach of Haza et al. [2008] by calculating the FSLEs for decreasing values of $\alpha(2,1.75$, $1.5,1.25,1.15$ ), chosing the smallest value (and thus the highest resolution in $\delta$ space) that does not contaminate the results when compared to those from larger values. We found that this minimum $\alpha$ depended upon the data density: for the dense CLIMODE array, the FSLEs for $\alpha=$ 1.15 agreed nearly perfectly with the coarser results from larger values. However, for the sparse ADB data set, values smaller than $\alpha=1.5$ altered the slope of the FSLEs at $\delta<30 \mathrm{~km}$ by spuriously increasing the values of $\lambda$ at smaller scales.

[63] Another less severe drawback to the FSLE approach is that the separation distance of a pair often does not grow 
monotonically across an interval $\left[\delta_{n}, \delta_{n+1}\right]$, and there is not a consensus on how to choose $\Delta t$ values in these cases. Some researchers (J. LaCasce, personal communication, 2009) choose the time of the first crossing of $\delta_{n}$ and of $\delta_{n+1}$ for each pair, so that there is one value of $\Delta t$ (and thus of $\lambda$ ) per pair, per distance bin (see Figure A1). Others (A. Haza and T. Özgökmen, personal communication, 2009) choose the fastest possible transitions from $\delta_{n}$ to $\delta_{n+1}$ (Figure A1). With this approach, if the pair separation crosses $\delta_{n}$ multiple times before subsequently crossing $\delta_{n+1}$, only the time of the final crossing of $\delta_{n}$ is used. In the fastest approach algorithm, multiple values may be derived for a pair, if it crossed from $\delta_{n}$ to $\delta_{n+1}$ multiple times. These two algorithms produce identical results for pairs which monotonically increase from $\delta_{n}$ to $\delta_{n+1}$. In practice, we found that these two algorithms produce similar results: $\lambda$ was consistently, albeit slightly, smaller by $\sim 0.88$ across all values of $\delta$ when using the first crossing algorithm, and the slope of $\lambda$ versus $\delta$ in a log-log plot was not sensitive to the choice of crossing algorithm. Note that this lack of sensitivity is true for $\left\langle 1 / \Delta t_{n}\right\rangle$, the ensemble average used to calculate the FSLEs, but not for the ensemble average $1 /\left\langle\Delta t_{n}\right\rangle$. The first crossing algorithm yields a $\Delta t_{n}$ distribution with more outliers at large values, compared to the fastest crossing algorithm. These large outliers are caused by the small number of pairs that spend an extended time crossing and recrossing $\delta_{n}$ before reaching $\delta_{n+1}$. They significantly increase the mean value $\left\langle\Delta t_{n}\right\rangle$ calculated via the first crossing algorithm, particularly at small values of $\delta$. In contrast, the fastest crossing algorithm produces many more estimates of $\Delta t_{n}$, and thus better resolves the distribution in a $\delta$ bin. As a consequence, the fastest crossing algorithm is far less sensitive to the distinction between $\left\langle 1 / \Delta t_{n}\right\rangle$ and $1 /\left\langle\Delta t_{n}\right\rangle$.

[64] In this study we used the fastest crossing algorithm for our FSLE calculations because it provides more degrees of freedom than the first crossing approach. We determined $95 \%$ confidence intervals for $\lambda_{n}$ by bootstrap resampling the $\Delta t$ values from all of the pairs in a given separation bin $\left[\delta_{n}, \delta_{n+1}\right]$.

[65] Acknowledgments. Dave Fratantoni and John Lund deployed many of the CLIMODE drifters described in this paper, and numerous individuals and organizations are responsible for the historical deployments. The authors had valuable discussions with numerous colleagues; interactions with Angelique Haza, Tamay Özgökmen, Shafer Smith, Joe LaCasce, Patrice Klein, John Toole, Kevin Speer, Carter Ohlmann, Javier Beron-Vera, Bill Dewer, and John Marshall were particularly enlightening. Mayra Pazos, Erik Vades, and Jessica Redman prepared the qualitycontrolled drifter data for this study. Shaun Dolk and Craig Engler managed worldwide drifter deployments and orchestrated the ADB drifter clusters. This work was supported by the National Science Foundation as part of the CLIMODE project. Additional support was provided by NOAA's Office of Climate Observations and the Atlantic Oceanographic and Meteorological Laboratory. The AVISO product was produced by the CLS Space Oceanography Division as part of the Environment and Climate EU ENACT project (EVK2-CT2001-00117) and with support from CNES. Microwave SST data are produced by Remote Sensing Systems and sponsored by the NASA Earth Science REASON DISCOVER Project and the AMSR-E Science Team. Data are available at http://www.remss.com.

\section{References}

Aref, H. (1984), Stirring by chaotic advection, J. Fluid Mech., 143, 1-21. Artale, V., G. Boffetta, A. Celani, M. Cencini, and A. Vulpiani (1997), Dispersion of passive tracers in closed basins: Beyond the diffusion coefficient, Phys. Fluids, 9, 115-173.
Aurell, E., G. Boffetta, A. Crisanti, G. Paladin, and A. Vulpiani (1997) Predictability in the large: An extension of the concept of Lyapunov exponent, J. Phys. A, 30, 1-26.

Bennett, A. F. (1984), Relative dispersion: Local and nonlocal dynamics, J. Atmos. Sci., 41, 1881-1886.

Bennett, A. F. (1987), A Lagrangian analysis of turbulent diffusion, Rev. Geophys., 25, 799-822.

Beron-Vera, F. J., M. J. Olascoaga, and G. J. Goni (2008), Oceanic mesoscale eddies as revealed by Lagrangian coherent structures, Geophys. Res. Lett., 35, L12603, doi:10.1029/2008GL033957.

Blumen, W. (1978), Uniform potential vorticity flow: Part I. Theory of wave interactions and two-dimensional turbulence, J. Atmos. Sci., 35, 774-783.

Capet, X., P. Klein, B. L. Hua, G. Lapeyre, and J. C. McWilliams (2008), Surface kinetic energy transfer in surface quasi-geostrophic flows, J. Fluid Mech., 604, 165-174.

Charney, J. G. (1971), Geostrophic turbulence, J. Atmos. Sci., 28, 1087-1095.

Chelton, D. B., R. A. deSzoeke, M. G. Schlax, K. El Naggar, and N. Siwertz (1998), Geographical variability of the first-barolinic Rossby radius of deformation, J. Phys. Oceanogr., 28, 433-460.

Corrsin, S. (1962), Theories of turbulent dispersion, in Méchanique de la Turbulence: Colloquium International CNRS, Marseilles, pp. 27-52, Cent. Natl. de la Rech. Sci., Paris.

Davis, R. (1982), On relating Eulerian and Lagrangian velocity statistics: Single particles in homogeneous flows, J. Fluid Mech., 114, 1-26.

Eldevik, T., and K. B. Dysthe (2002), Spiral eddies, J. Phys. Oceanogr., 32, 851-869.

Elipot, S., and R. Lumpkin (2008), Spectral description of near-surface variability, Geophys. Res. Lett., 35, L05606, doi:10.1029/ 2007GL032874

Er-el, J., and R. L. Peskin (1981), Relative diffusion of constant-level balloons in the Southern Hemisphere, J. Atmos. Sci., 38, 2264-2274

Flament, P. J., R. Lumpkin, J. Tournadre, R. Kloosterziel, and L. Armi (2001), Vortex pairing in an unstable anticyclonic shear flow: discrete subharmonics of one pendulum day, J. Fluid Mech., 440, 401-410.

Fu, L.-L., and R. Ferrari (2008), Observing oceanic submesoscale processes from space, Eos Trans. AGU, 89(48), 488-489.

Griffa, A., R. Lumpkin, and M. Veneziani (2008), Cyclonic and anticyclonic motion in the upper ocean, Geophys. Res. Lett., 35, L01608, doi:10.1029/2007GL032100.

Haza, A. C., A. C. Poje, T. M. Özgökmen, and P. Martin (2008), Relative dispersion from a high-resolution coastal model of the Adriatic sea, Ocean Modell., 22, 48-65, doi:10.1016/j.ocemod.2008.01.006.

Isern-Fontanet, J., B. Chapron, G. Lapeyre, and P. Klein (2006), Potential use of microwave sea surface temperatures for the estimation of ocean currents, Geophys. Res. Lett., 33, L24608, doi:10.1029/ 2006GL027801.

Isern-Fontanet, J., G. Lapeyre, P. Klein, B. Chapron, and M. Hecht (2008), Three-dimensional reconstruction of oceanic mesoscale currents from surface information, J. Geophys. Res., 113, C09005, doi:10.1029/ 2007JC004692.

Klein, P., S. L. Smith, and G. Lapeyre (2003), Organization of near-inertial energy by an eddy field, Q. J. R. Meteorol. Soc., 130, 1153-1166.

Klein, P., B. L. Hua, G. Lapeyre, X. Capet, S. Le Gentil, and H. Sasaki (2008), Upper ocean turbulence from high-resolution 3D simulations, J. Phys. Oceanogr., 38, 1748-1763.

Kolmogorov, A. N. (1941), The local structure of turbulence in incompressible viscous fluid for very large reynolds numbers, Dokl. Akad. Nauk SSSR, 30, 299-303.

Koszalka, I., J. H. LaCasce, and K. A. Orvik (2009), Relative dispersion in the Nordic Seas, J. Mar. Res., 67, 411-433.

Kraichnan, R. H. (1967), Inertial ranges of two dimensional turbulenct, Phys. Fluids, 10, 1417-1423.

LaCasce, J. H. (2008), Statistics from Lagrangian observations, Prog Oceanogr, 77, 1-29.

LaCasce, J. H., and A. Bower (2000), Relative dispersion in the subsurface North Atlantic, J. Mar. Res., 58, 863-894.

LaCasce, J. H., and J. C. Ohlmann (2003), Relative dispersion at the surface of the Gulf of Mexico, J. Mar. Res., 61, 285-312.

Lapeyre, G. (2009), What vertical mode does the altimeter reflect? On the decomposition in baroclinic modes and on a surface-trapped mode, J. Phys. Oceanogr., 39, 2857-2874.

Lapeyre, G., and P. Klein (2006), Dynamics of the upper oceanic layers in terms of surface quasigeostrophic theory, J. Phys. Oceanogr., 36, $165-176$.

Le Traon, P., F. Nadal, and N. Ducet (1998), An improved mapping method of multisatellite altimeter data, J. Atmos. Oceanic Technol., 15, $522-534$. 
Le Traon, P., P. Klein, and B. L. Hua (2008), Do altimeter spectra agree with the interior or surface quasigeostrophic theory?, J. Phys. Oceanogr., $38,1137-1142$.

Lumpkin, R., and M. Pazos (2007), Measuring surface currents with Surface Velocity Program drifters: The instrument, its data and some recent results, in Lagrangian Analysis and Prediction of Coastal and Ocean Dynamics, edited by A. Griffa et al., chap. 2, pp. 39-67, Cambridge Univ. Press, Cambridge, U. K.

Lumpkin, R., A.-M. Treguier, and K. Speer (2002), Lagrangian eddy scales in the northern Atlantic Ocean, J. Phys. Oceanogr., 32, 2425-2440.

Marshall, J., et al. (2009), The CLIMODE field campaign: Observing the cycle of convection and restratification over the Gulf Stream, Bull. Am. Meteorol. Soc., 90, 1337-1350, doi:10.1175/2009BAMS2706.1.

McWilliams, J. C. (1985), Submesoscale, coherent vortices in the ocean, Rev. Geophys., 23, 165-182.

Morel, P., and M. Larcheveque (1974), Relative dispersion of constantlevel balloons in the $200 \mathrm{mb}$ general circulation, J. Atmos. Sci., 31, 2189-2196.

Munk, W., L. Armi, K. Fischer, and F. Zachariasen (2000), Spirals on the sea, Proc. R. Soc. London A., 456, 1217-1280.

Niiler, P. P. (2001), The world ocean surface circulation, in Ocean Circulation and Climate: Observing and Modelling the Global Ocean, Int. Geophys. Ser., vol. 77, edited by G. Siedler, J. Church, and J. Gould, pp. 193-204, Academic, San Diego, Calif.

Niiler, P. P., and J. D. Paduan (1995), Wind-driven motions in the northeast Pacific as measured by Lagrangian drifters, J. Phys. Oceanogr., 25, 2819-2830.

Niiler, P. P., R. Davis, and H. White (1987), Water-following characteristics of a mixed-layer drifter, Deep Sea Res. Part A, 34, 1867-1882.

Ollitrault, M., C. Gabillet, and A. C. de Verdière (2005), Open ocean regimes of relative dispersion, J. Fluid Mech., 533, 381-407.

Provenzale, A. (1999), Transport by coherent barotropic vortices, Annu. Rev. Fluid Mech., 31, 55-93.

Richardson, L. F. (1926), Atmospheric diffusion on a distance-neighbour graph, Proc. R. Soc. London A, 110, 709-737.

Richardson, L. F., and H. Stommel (1948), Note on eddy diffusion in the sea, J. Meteorol., 5, 238-240.
Rio, M.-H., and F. Hernandez (2004), A mean dynamic topography computed over the world ocean from altimetry, in situ measurements, and a geoid model, J. Geophys. Res., 109, C12032, doi:10.1029/ 2003JC002226.

Rudnick, D. L. (2001), On the skewness of vorticity in the upper ocean, Geophys. Res. Lett., 28, 2045-2048.

Shen, C. Y., and T. E. Evans (2002), Inertial instability and sea spirals, Geophys. Res. Lett., 29(23), 2124, doi:10.1029/2002GL015701.

Taylor, G. (1921), Diffusion by continuous movements, Proc. London Math. Soc., 20, 196-212.

Thomas, L. N., A. Tandon, and A. Mahadevan (2008), Submesoscale processes and dynamics, in Ocean Modeling in an Eddying Regime, Geophys. Monogr. Ser., vol. 177, edited by M. Hect and H. Hasume, pp. 17-38, AGU, Washington, D. C.

Tulloch, R. T., and K. S. Smith (2006), A theory for the atmospheric energy spectrum: Depth-limited temperature anomalies at the tropopause, Proc Natl. Acad. Sci. U. S. A., 103, 14,690-14,694.

Tulloch, R. T., and K. S. Smith (2009), Quasigeostrophic turbulence with explicit surface dynamics: Application to the atmospheric energy spectrum, J. Atmos. Sci., 66, 450-467.

Wang, D.-P., C. N. Flagg, K. Donohue, and H. T. Rossby (2010), Wavenumber spectrum in the Gulf Stream from shipboard ADCP observations and comparisons with altimetry measurements, J. Phys. Oceanogr., 40, 840-844.

Waugh, D. W., and E. R. Abraham (2008), Stirring in the global surface ocean, Geophys. Res. Lett., 35, L20605, doi:10.1029/2008GL035526.

Zhurbas, V., and I. S. Oh (2004), Drifter-derived maps of lateral diffusivity in the Pacific and Atlantic oceans in relation to surface circulation patterns, J. Geophys. Res., 109, C05015, doi:10.1029/2003JC002241.

S. Elipot, National Oceanography Centre, Joseph Proudman Building, 6 Brownlow St., Liverpool L3 5DA, UK.

R. Lumpkin, NOAA Atlantic Oceanographic and Meteorological Laboratory, 4301 Rickenbacker Cswy., Miami, FL 33149, USA. (rick.lumpkin@noaa.gov) 